\title{
An optimal control approach to scheduling maintenance and production in parallel lines of reactors using decaying catalysts
}

\author{
S. D. Adloor ${ }^{\mathrm{a}, *}$, V. S. Vassiliadis ${ }^{\mathrm{b}}$ \\ ${ }^{a}$ Department of Chemical Engineering and Biotechnology, University of Cambridge, \\ Philippa Fawcett Drive, Cambridge, CB3 0AS, United Kingdom \\ ${ }^{b}$ Cambridge Simulation Solutions Ltd., 8 Cody Road, Waterbeach, Cambridge, CB25 \\ gLS, United Kingdom
}

\begin{abstract}
In this work, an optimal control approach for scheduling maintenance and production in a process using decaying catalysts, originally developed in a previous study for a single reactor, is extended to parallel lines of reactors. Unlike traditional mixed-integer optimisation methods, this approach involves formulating this problem as a multistage mixed-integer optimal control problem (MSMIOCP) and using a methodology that enables solution as a standard nonlinear optimisation problem. The methodology's features of a feasible path approach and scheduling catalyst changeovers without combinatorial optimisation techniques can be advantageous in providing reliable, robust and efficient solutions in comparison to mixed-integer methods. The MSMIOCP formulation is applied to the case study of an industrial process that operates a single feed over a set of 4 parallel reactors and produces a single product. The solutions obtained using the proposed methodology are of high quality and highlight the potential advantages of this approach over mixed-integer techniques.
\end{abstract}

Keywords: Optimal control problem; Mixed-integer Optimisation; Catalyst

\footnotetext{
*Corresponding author

Email address: sa814@cam.ac.uk (S. D. Adloor)
} 
replacement; Parallel lines; Scheduling; Production planning

\section{Introduction and literature review}

The phenomenon of catalyst deactivation presents significant economic challenges to industries. Catalyst deactivation is inevitable and the decaying performance leads to a reduction in product yield, which in turn could lead to an inability to meet product demand and hence a loss in revenue.

To restore process performance and improve on low product yields, a maintenance action is required which involves shutting down the reactor using the decayed catalyst and replacing the decayed catalyst with a fresh catalyst that has full activity. Such a maintenance action is called a catalyst replacement or a catalyst changeover operation. While this maintenance action does improve product yield, there are negative impacts associated with this operation such as a loss of production time because of the reactor being shut down and the energy and labour costs to replace the catalyst.

In order to avoid stopping production completely, industries commonly use parallel processing lines to manufacture products. A parallel set up can improve the flexibility of the production process by allowing one reactor to be shut down for catalyst replacement while the remaining reactors continue to produce product to meet demand.

However, in order to ensure efficient operation in such a set up, there is a trade-off to be addressed for each reactor: while frequently renewing catalyst loads results in a high production rate, it also leads to large maintenance costs and loss in production occurring from the process shut-down for catalyst changeovers. This trade-off can be optimally managed by the development of a maintenance schedule that specifies the optimum number of catalyst loads to use and the optimal time for catalyst replacement in each 
reactor of the parallel set up, over a fixed time horizon. The maintenance schedule may also be required to fulfil a constraint that no two reactors undergo catalyst replacement at the same time due to production requirements or the maintenance labour and equipment availability.

Apart from obtaining such a maintenance schedule, it is also necessary to identify the optimal operating conditions, such as the flow rate to and temperature of operation of each reactor during the times the catalyst is in operation, while also taking into account the catalyst deactivation. In addition, the maintenance schedule and operating conditions should be tailored to produce an adequate inventory of product that enables sales to effectively meet varying demand across the time horizon, while also avoiding excessively high storage costs. An integrated execution of all of these decisions in an optimal manner can greatly minimise the negative effects of catalyst deactivation, and thereby maximise the profits of the process. However, such an execution requires solving a highly challenging modelling and optimisation problem containing a very large number of variables and constraints.

A thorough literature survey did not result in finding any work that has explicitly claimed to optimise maintenance scheduling, operating conditions, inventory management and sales to meet time-varying demand involving parallel lines of reactors using decaying catalysts. While recently, there has been work for production scheduling involving catalytic reactors using economic model predictive control (e.g. Alanqar et al. (2016, 2017), Ellis et al. (2017)), the aforementioned aspects have not been explored. However, there has been considerable work investigating the integrated optimisation of maintenance scheduling and production in different industrial applications which use parallel processing lines that experience varied types of decaying performances.

For example, the integrated optimisation of the maintenance scheduling 
of parallel lines and sales to meet time-varying demand has been considered in works by Castro et al. (2014) for a gas engine plant and by Liu et al. (2014) in the bio-pharmaceutical industry while considering the decaying performance of a chromatography resin. But these works did not consider the optimisation of nonlinear operating conditions, and so, less computationally intensive Mixed Integer Linear Programming (MILP) models could be used for the solution of these problems.

For a parallel network of compressors experiencing decaying performance due to fouling and degradation by fluid particles, Kopanos et al. (2015) and Xenos et al. (2016) have optimised the maintenance scheduling, the operating flow rate and inventory management to meet varying demand. But the authors admit to seeking to avoid hard Mixed Integer Nonlinear Programming (MINLP) formulations by linearising equations to form MILP models and concede that such linear approximations can cause errors in the results.

Heluane et al. $(2004,2007)$ have developed MINLP formulations to optimise the maintenance scheduling and operating flow rates of parallel evaporator systems that decay in performance due to heat transfer induced fouling. However, these works focused on obtaining cyclic schedules and did not consider the problem of inventory management or sales to meet seasonal demand.

In petrochemical plants, a cracking furnace is used to break long chain hydrocarbons into valuable products such as ethylene and these plants have multiple such furnaces operating in parallel. Coke depositions on the walls reduce the efficiency of the furnaces and maintenance actions for decoking operations are necessary to restore performance. In tandem with these decoking operations, other considerations may need to be managed such as the operating conditions of flow rates to and temperature of the furnace, as well as product inventory management to meet seasonal demand. A number of 
strategies have been developed to manage some or all of these aspects.

Most of the studies undertaken have focused on developing cyclic schedules based on MINLP methodologies where only decoking schedules and operating conditions have to be optimised, while assuming a constant product demand (or sales) over an infinite time horizon. These include works by Jain and Grossmann (1998), Liu et al. (2010), Zhao et al. (2010) and Jin et al. (2015). However, these works face shortcomings: only Liu et al. (2010) and Zhao et al. (2010) obtain schedules without simultaneous decoking and only Jin et al. (2015) obtains dynamic operational profiles. Another work by Lim et al. (2006) has considered a fixed time horizon rather than a cyclic schedule but the demand considered is constant and the solution methodology involves treating integer and continuous variables separately, which can be inadequate if those variables are highly interdependent.

As traditional gradient based methods can face difficulties in handling MINLP models with highly nonlinear constraints, population based optimisation methods have also been attempted to obtain such cyclic schedules. For example, Yu et al. (2017) have used a Diversity Learning Teaching Learning Based Optimisation (DLTLBO) algorithm to solve such a problem. Lin and $\mathrm{Du}$ (2018) have proposed a two-level nested formulation for this problem which uses a Genetic Algorithm to solve the outer MILP problem and a Sequential Quadratic Programming algorithm to solve the inner NLP problem. Though solutions obtained were better than from MINLP techniques, optimality could not be guaranteed as metaheuristic approaches are involved.

Only a limited set of publications consider the optimisation of all aspects of decoking scheduling, operating conditions as well as inventory management and sales to meet time-varying demand. These are discussed next. 
Schulz et al. (2006a,b) have developed a multistage MINLP model for optimising all these aspects in an ethylene plant operating 8 furnaces in parallel. However, their work did not reveal the underlying model equations and had restrictive assumptions such as identical cycle times for all furnaces and a linear coking rate.

Su et al. (2016) have considered optimisation of all these aspects using a hybrid MINLP algorithm developed in Su et al. (2015) that enabled faster convergence in large scale problems compared to standard MINLP methods. However, the model involved simplistic assumptions such as a linear coking rate and constant operating flow rates. More complex models that could cause greater intractability of the MINLPs have not been investigated.

Finally, Wang et al. (2016) have proposed a Lagrangian decomposition method to solve such a problem. While the algorithm has produced better solutions in comparison to standard MINLP solvers, the authors admit that due to the complexity of the formulation, they face difficulties in converging to optimality. Further, this method can mainly be applied only in cases where the underlying model exhibits a block angular structure.

The preceding literature review indicates that few papers address the entirety of the problem of optimising the maintenance scheduling of parallel processing lines that experience decaying performance in combination with operational planning, inventory management and sales to meet time-varying demand. These articles report difficulties in attaining optimality, even after applying significant approximation and decomposition techniques, thereby indicating the complexity of the problem. Even the other publications that address only a subset of these decisions exhibit such shortcomings.

These difficulties can be traced to the mixed-integer formulations of these 
problems. The combinatorial nature of these solution methods causes the problem to become intractable in terms of size and solution times. In addition, these methods attempt to circumvent differential equations and other nonlinear terms using linear approximation techniques, which besides reducing solution accuracy, also increases the number of variables and constraints involved. More complex models or an increase in the scale of the problem and the number of decisions can further accentuate these difficulties.

To overcome these difficulties, a shift away from mixed-integer optimisation approaches is needed. While population-based algorithms have been suggested, as metaheuristic techniques, they cannot guarantee a theoretical convergence to optimality. A methodology is needed that can provide a reliable, robust and efficient solution to the problem. And this should be possible even for large scale problems, regardless of the degree of nonlinearity of the underlying equations.

A previous work by Adloor et al. (2020) developed an optimal control (dynamic optimisation) methodology for scheduling maintenance and production in a process using decaying catalysts, for the case of a single reactor. The methodology produced reliable, robust and efficient solutions in comparison to mixed-integer techniques. In this paper, that methodology is applied to a process containing parallel lines of reactors using decaying catalysts. The main contributions of this paper lie in demonstrating the effectiveness of the optimal control methodology in optimising the maintenance scheduling of the parallel set up in combination with the operational planning, inventory management and sales to meet seasonal demand. In fact, this methodology is applicable to any of the previously mentioned applications involving maintenance scheduling of parallel processing lines experiencing decaying performances. 
This brings to an end, Section 1, which forms the introductory and literary review part of the paper. The rest of the paper is organised as follows. In Section 2, the optimal control formulation of this problem is presented and the solution methodology is detailed in Section 3. In Section 4, the optimal control formulation is applied to the case study of an industrial process and the results obtained are discussed. The conclusions of the paper and other notable points are discussed in Section 5. Appendix A contains a table providing supplementary information.

\section{The optimal control problem formulation}

In this section, an optimal control formulation is presented for the problem of optimising maintenance scheduling and production planning in a parallel set of reactors containing decaying catalysts. This formulation is characterised by a set of decision (or control) and state variables. The decisions here include those of when to schedule a maintenance action to replace the catalyst in each reactor, which are binary in nature, as well as those that decide the operating conditions for each reactor and the sales, which are continuous variables. The state variables represent the 'state' of the process and are determined by the values of the decision variables, a set of appropriate Ordinary Differential Equations (ODEs) and constraints.

The basic formulation of an optimal control problem (OCP) is shown in equations (1a) - (1g). The performance index consists of a point index $\phi$ and a continuous index $L$. This performance index is minimised by the selection of controls, $w(t)$, and differential state variables, $x(t)$, subject to differential equations, $h$ and constraints, $c$. Equations (1b) - (1c) describe an ODE system, given fixed initial and final times, $t_{0}$ and $t_{F}$, respectively, and initial condition $x_{0}$. The controls $w(t)$ can include binary controls, $u(t)$, as well as continuous controls, $v(t)$, that belong to a real permissible set $\mathcal{V}$. 


$$
\min _{w(t)} W=\phi\left(x\left(t_{F}\right)\right)+\int_{t_{0}}^{t_{F}} L(x(t), w(t), t) \mathrm{d} t
$$

subject to

$$
\begin{gathered}
\dot{x}(t)=h(x(t), w(t), t) \\
\forall t \in\left[t_{0}, t_{F}\right] \\
x\left(t_{0}\right)=x_{0} \\
c(x(t), w(t), t) \leq 0 \\
\forall t \in\left[t_{0}, t_{F}\right] \\
w(t)=\left[[u(t)]^{T},[v(t)]^{T}\right]^{T} \\
u(t) \in\{0,1\} \\
v(t) \in \mathcal{V}
\end{gathered}
$$

In order to apply the OCP formulation to the problem under consideration, the whole time horizon of the process is discretised into stages, which can be of arbitrary length. A control parametrisation approach is adopted wherein the decision variables are discretised over the whole time horizon and are taken to be piecewise constant across the times corresponding to each stage. That is, if the total number of stages is $N P$, the collective vectors of the controls, $u$ and $v$, take up the following form:

$$
\begin{aligned}
& u=\left[u^{(1)}, u^{(2)}, \ldots, u^{(N P)}\right]^{T} \\
& v=\left[v^{(1)}, v^{(2)}, \ldots, v^{(N P)}\right]^{T}
\end{aligned}
$$

The control profiles are allowed to be discontinuous at the junctions, $t_{p}$, between any two consecutive stages, $p$ and $p+1$.

The state variables, however, are retained in their continuous form, with- 
out discretisation, and are determined in each stage from a set of ODEs. The ODEs are solved to a high accuracy in the right sequential order using state-of-the-art integrators and hence, this solution methodology is called a "feasible path approach" (Vassiliadis, 1993; Vassiliadis et al., 1994a,b). The solutions of the ODEs in each stage, across the whole time horizon, are facilitated by junction conditions between any two consecutive periods, $p$ and $p+1$, the general form of which is given by equation (3) (Vassiliadis, 1993):

$$
\begin{aligned}
& J\left(\dot{x}^{(p+1)}\left(t_{p}^{+}\right), x^{(p+1)}\left(t_{p}^{+}\right), u^{(p+1)}\left(t_{p}^{+}\right), v^{(p+1)}\left(t_{p}^{+}\right),\right. \\
&\left.\dot{x}^{(p)}\left(t_{p}^{-}\right), x^{(p)}\left(t_{p}^{-}\right), u^{(p)}\left(t_{p}^{-}\right), v^{(p)}\left(t_{p}^{-}\right), t_{p}\right)=0 \\
& p=1,2, \ldots N P-1
\end{aligned}
$$

The discretisation of the time horizon into multiple stages and the presence of integer and continuous decision variables leads the OCP to take up a Multistage Mixed-Integer Optimal Control Problem (MSMIOCP) form. The basic form of the MSMIOCP over time periods, $p=1,2, \ldots N P$, $t \in\left[t_{p-1}, t_{p}\right]$, with $t_{N P}=t_{F}$ is shown in equations (4a) - (4g).

$\min _{u, v} W=\sum_{p=1}^{N P}\left\{\phi^{(p)}\left(x^{(p)}\left(t_{p}\right), u^{(p)}, v^{(p)}, t_{p}\right)+\int_{t_{p-1}}^{t_{p}} L^{(p)}\left(x^{(p)}(t), u^{(p)}, v^{(p)}, t\right) \mathrm{d} t\right\}$

subject to

$$
\begin{gathered}
\dot{x}^{(p)}(t)=h^{(p)}\left(x^{(p)}(t), u^{(p)}, v^{(p)}, t\right) \\
t_{p-1} \leq t \leq t_{p} \\
p=1,2, \ldots, N P \\
x^{(1)}\left(t_{0}\right)=g^{(1)}\left(u^{(1)}, v^{(1)}\right)
\end{gathered}
$$




$$
\begin{gathered}
x^{(p)}\left(t_{p-1}\right)=g^{(p)}\left(x^{(p-1)}\left(t_{p-1}\right), u^{(p)}, v^{(p)}\right) \\
p=2,3, \ldots, N P \\
c^{(p)}\left(x^{(p)}(t), u^{(p)}, v^{(p)}, t\right) \leq 0 \\
t_{p-1} \leq t \leq t_{p} \\
p=1,2, \ldots, N P \\
u^{(p)} \in\{0,1\} \\
p=1,2, \ldots, N P \\
v^{(p)} \in \mathcal{V} \\
p=1,2, \ldots, N P
\end{gathered}
$$

In the above equation, the terminology used is similar to the basic OCP formulation, with the superscript $(p)$ indicating that they apply to stage, $p$. The additional terms here are the junction conditions, $g$, analogous to equation (3), that provide the initial condition for the solution of the ODEs in stage $p$. An illustration of the MSMIOCP formulation is shown in Figure 1.

In this multistage formulation, in each stage, for each reactor, a decision has to be made on whether the catalyst should be in operation or a maintenance action should occur to replace it. This decision is of binary nature and hence, corresponds to the controls, $u$. Henceforth in this article, this binary decision will be referred to as a 'catalyst changeover control'. Further, the plant operating conditions of flow rate to and temperature of operation of each reactor and the amount of product sales should also be decided at each stage, which are decisions of continuous form and so, correspond to the controls, $v$. Due to the presence of integer and continuous controls, this is a mixed-integer formulation.

But as mentioned in Section 1, mixed-integer optimisation techniques suffer from drawbacks. So, in the next section, a methodology is presented 


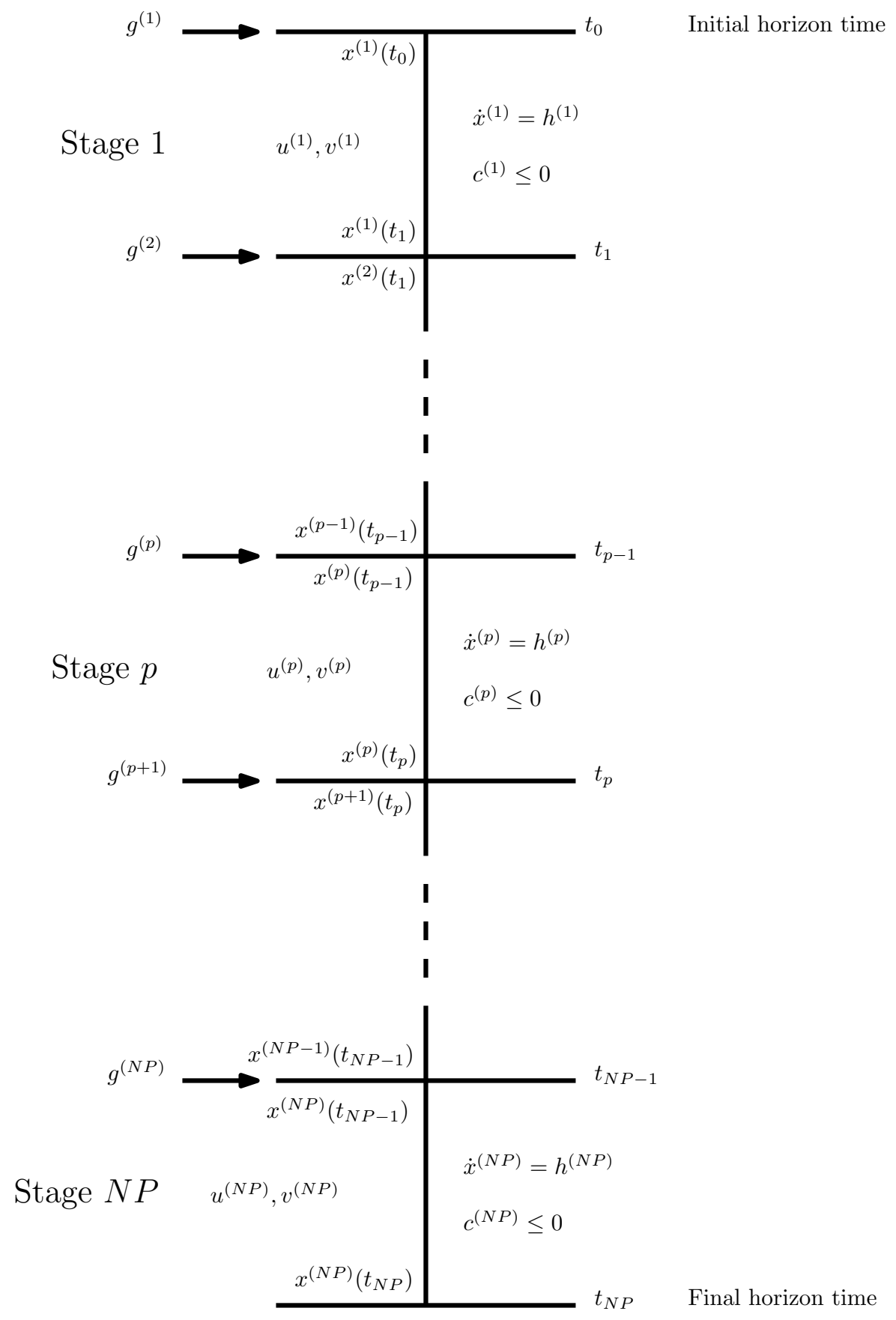

Figure 1: An illustration of the MSMIOCP formulation 
that enables a solution of this problem as a standard nonlinear optimisation problem, which obviates the use of mixed-integer optimisation methods.

\section{Problem solution methodology}

In work of Adloor et al. (2020), two solution methodologies were presented for solving an MSMIOCP formulation, of the problem of scheduling maintenance and production in a single reactor using decaying catalysts, as a standard nonlinear optimisation problem.

The first methodology, titled Implementation I, demonstrated a theoretically interesting property of bang-bang behaviour for the binary catalyst changeover controls. But it could not handle highly nonlinear models and even in the less nonlinear models, it had a tendency of converging prematurely or crashing due to integration problems depending on the initial guesses used.

However, the second methodology, Implementation II, while not demonstrating the bang-bang behaviour, was very successful in providing reliable, robust and efficient solutions, regardless of the nonlinearity of the equations involved or the initial guesses supplied. Further, the optimality of the solutions obtained from Implementation II were justified by the good correlation with the bang-bang solutions of Implementation I, which were theoretically proven to be optimal.

Therefore, Implementation II will be used in this paper. For the sake of completeness, the principle behind the solution methodology of Implementation II is presented once again in this section.

In order to execute this solution methodology, the binary nature of the controls, $u^{(p)}$ for stage $p$, in equation (4f), is relaxed and they are instead 
considered as continuous variables that vary between 0 and 1 . That is:

$$
\begin{gathered}
u^{(p)} \in[0,1] \\
p=1,2, \ldots, N P
\end{gathered}
$$

Thus now, only a standard multistage optimal control problem (MSOCP), free from binary variables, has to be solved using the feasible path approach, which can be done using any nonlinear optimisation algorithm. The requirement for the controls, $u^{(p)}$, for stage $p$, to take 0 or 1 values is enforced using a penalty term homotopy technique, similar to that suggested by Sager (2005, 2009). In this technique, a monotonically increasing penalty term is added to the objective function in equation (4a) and a series of MSOCPs of the following generic form are solved:

$$
F_{k}: \min \left[W+M_{k} \sum_{p=1}^{N P} u^{(p)}\left[1-u^{(p)}\right]\right]
$$

subject to equations $(4 \mathrm{~b})-(4 \mathrm{e}),(5)$ and $(4 \mathrm{~g})$, for

$$
\begin{gathered}
k=1,2,3 \ldots \\
M_{1}=0
\end{gathered}
$$

Every iteration, $k$, is referred to as 'major iteration'. The first major iteration $(k=1)$ of the series is designated a weight of $M_{1}=0$ and is similar to solving the problem given by equation (4), the only difference being there are no integer restrictions on controls $u$. If solving problem $F_{1}$ does not produce binary values for controls $u$, the second major iteration occurs in which a weight $M_{2}>0$ is chosen and problem $F_{2}$ is solved using the solution of $F_{1}$ as initial guesses. This procedure is repeated in an iterative manner, by choosing a weight $M_{k+1}>M_{k}$ and solving problem $F_{k+1}$ with the solution of $F_{k}$ as initial guesses, until iteration $K(K \geq 1)$ such that all controls in $u$, in 
the solution of problem $F_{K}$, are forced by weight $M_{K}$ to take values of either 0 or 1 . The solution methodology is represented as an algorithmic flowchart in Figure 2.

The progression for the increase of weights, $M_{k}$, is chosen arbitrarily, by trial and error, and is dependent on the parameters of the problem. It should be remembered that if the weight is increased too slowly, the computational time becomes large, while if it is increased too fast, the optimiser can fail to recognise a solution and continue iterations indefinitely.

The proposed solution methodology, as a standard multistage optimal control problem using a feasible path approach, can provide a number of advantages over combinatorial optimisation techniques:

1. The feasible path approach employs state-of-the-art integrators which can solve nonlinear differential and algebraic equations to a high accuracy. Thus, the solutions obtained are expected to be more reliable than in the mixed-integer formulations which solve such equations using linear approximation techniques.

2. The linear approximation techniques used by mixed-integer formulations to handle the differential equations and nonlinear terms, often cause the problem to end up containing a very large number of variables and constraints. This leads to difficulties in convergence to optimal solutions. However, in the feasible path approach, the differential equations and nonlinear terms are solved by an integrator without creating additional variables or constraints to be considered in the optimisation phase. The optimisation problem is of much smaller size with a few continuous only decision variables and constraints, and obtaining solutions is well within the scope of existing NLP solvers, regardless of the initial guesses used. Hence, the methodology is expected to be more robust in comparison to the combinatorial optimisation techniques. 


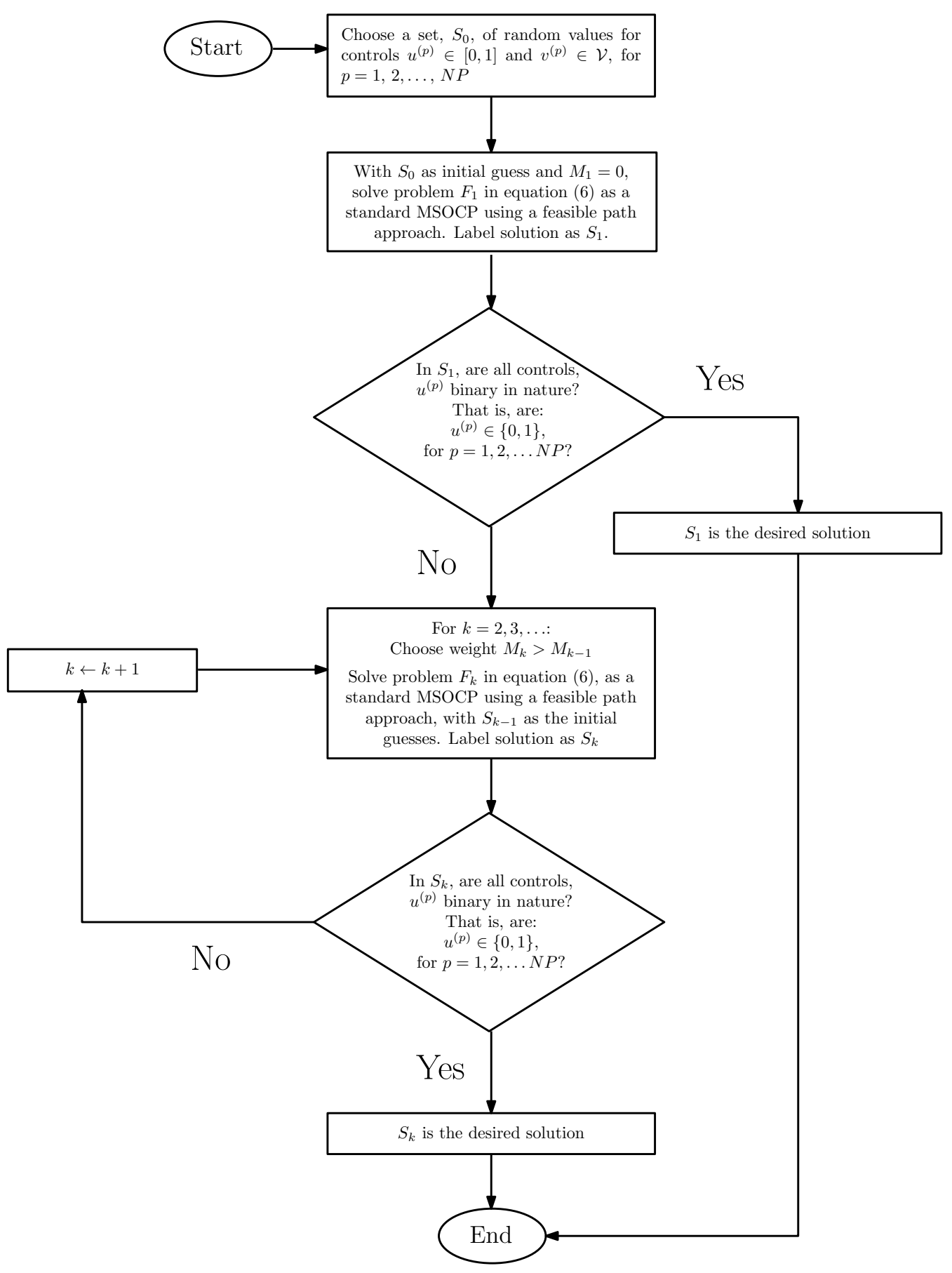

Figure 2: An algorithmic flowchart for the solution methodology 
3. By virtue of the penalty term homotopy technique, a weight term in the objective function forces the controls, originally binary but considered continuous in this formulation, to take values of either 0 or 1 . Thus, the 0 or 1 values for these controls, which correspond to the catalyst changeover actions, are decided inherently during the optimisation, without using mixed-integer techniques. Hence, no additional computational effort is spent in deciding when to schedule catalyst changeovers, thereby underlining the potential efficiency of this methodology over combinatorial optimisation approaches.

A drawback of the feasible path approach is the high computational effort spent in solving the differential equations at each iteration of the optimisation. However, with the advent of high performance computing and parallel computing facilities, the methodology's feature of scalability of the problem can be exploited. That is, once decision variables are fixed at each iteration of the optimisation, each reactor can be simulated entirely separately on a different computer. Furthermore, any required gradient evaluations can be further parallelised within the computer on which each reactor's simulation occurs.

Another limitation is that solution as an MSOCP leads to the problem becoming non-convex, as it is does not involve any binary/integer decisions. A local solution is inevitable due to this non-convexity, but it is now far easier to use practical approaches to (a) explore a further improvement of local solutions, e.g. by multi-start methods, and (b) it is important to note that a placement of a maintenance action up or down in the planning horizon by a couple of periods (e.g. weekly planning periods) has little impact, sensitivitywise, to a long planning horizon problem. For industrial application, global optimality in such cases may have little value in itself, due to factors such as disturbances, uncertainties and inaccuracies in the overall process model, which in this light gives a serious advantage to the methodology adopted and 
put forward in this work.

The preceding discussion indicates that this solution methodology has great potential for providing reliable, robust and efficient solutions to the problem under consideration, in comparison to mixed-integer techniques. In the next section, the effectiveness of the MSOCP formulation is demonstrated by an application to the case study of an industrial process.

\section{Case Study: Problem formulation, implementation, results and discussion}

In this section, the MSMIOCP formulation is applied in a case study to optimise maintenance scheduling, operation and sales to meet time-varying demand in an industrial process wherein a single feed is split over a set of parallel reactors using decaying catalysts to produce a single product. As mentioned in Section 1, currently no publication explicitly addresses such a problem. A work by Schulz et al. (2006a) that focused on optimising a similar set up in an ethylene plant using MINLP approaches does not reveal the underlying process equations. Hence, there was no process in any publication that could be used as a base to develop this formulation. Instead, the process considered in Adloor et al. (2020), for a single reactor, is modified to consider 4 parallel reactors.

A schematic of the process is shown in Figure 3. Here, a maintenance schedule is required that specifies for the set of parallel reactors, how many catalyst loads to use in each reactor as well as when the maintenance action to replace each of the used catalyst should occur in each reactor. In addition, for each reactor in the parallel set up, a production plan is needed that specifies the flow rate to and temperature of operation of each reactor at all times, as well as the periodic sales to meet time-varying demand. This production plan should be managed in tandem with the maintenance schedule and while 
taking catalyst deactivation in each reactor into account.

The objective is to maximise the profits of the process by an integrated optimisation of the maintenance scheduling and production operations in the set of parallel reactors that use decaying catalysts. The essential elements of the problem formulation and the implementation details are discussed before presenting the results obtained.

\subsection{Problem formulation}

In the problem addressed, the following assumptions apply:

1. The industrial process operates over a fixed time horizon, in the order of years. Each year is constituted by 12 months and there are a total of $N M$ months, wherein each month is constituted by 4 weeks.

2. The industrial process functions according to a certain process model and is subject to operating constraints.

3. The process has 4 Continuous Stirred Tank Reactors (CSTRs) of equal volumes, that operate in parallel.

4. There is a single feed to the process which is to be divided among the 4 reactors.

5. Each reactor processes the inlet feed using a catalyst to manufacture the same type of product.

6. In all reactors, the catalyst performance decays with time and has to be replaced before it crosses a certain maximum age.

7. The catalyst deactivation kinetics is first order with respect to the catalyst activity and is independent of the concentration of the reacting species. That is, the deactivation rate equation is of general form: 


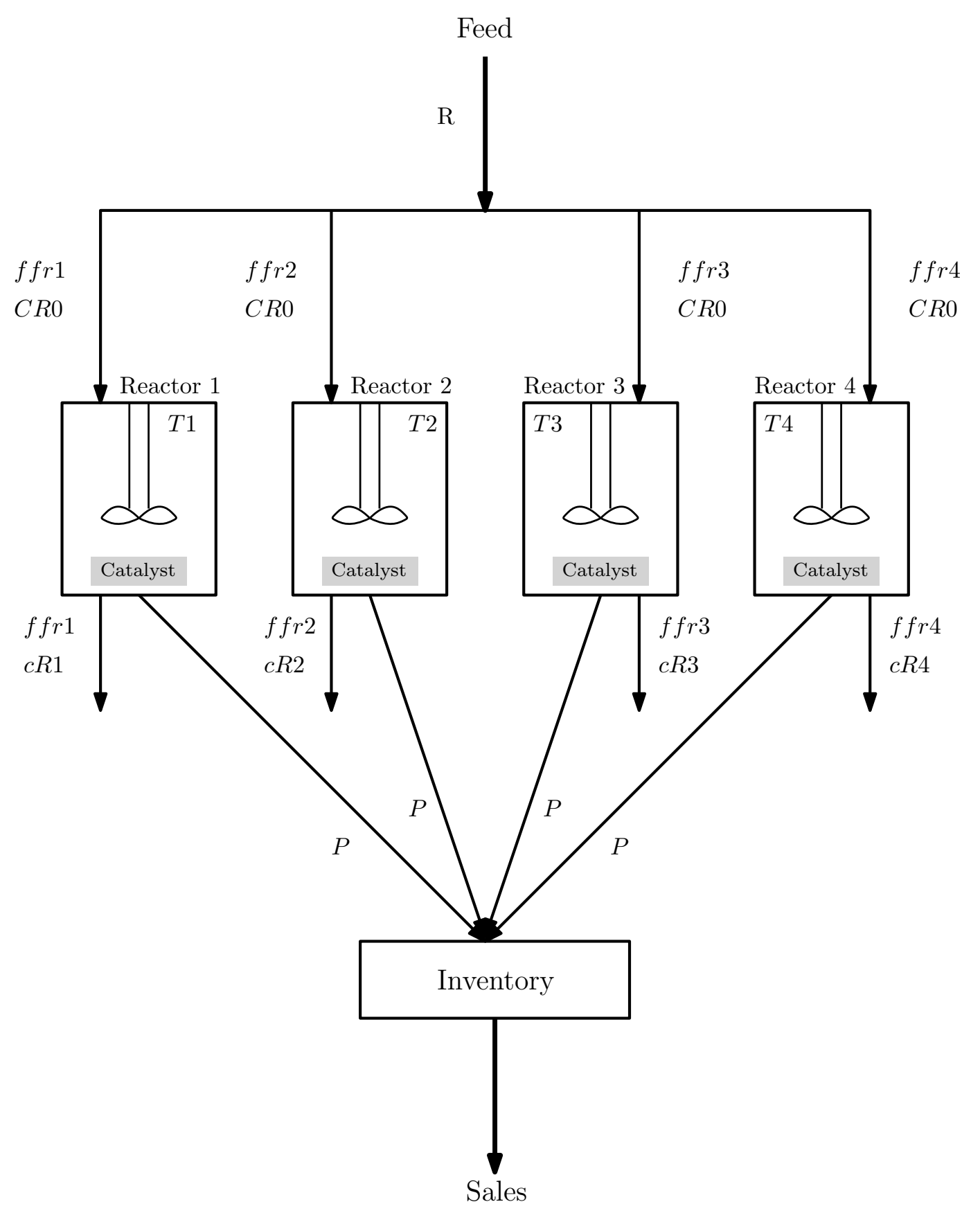

Figure 3: A schematic of the process 


$$
\frac{d\left(c a t_{-} a c t\right)}{d t}=-K_{d} \times c a t_{-} a c t
$$

where $K_{d}$ is the deactivation rate constant and cat_act is the activity of the catalyst.

8. The rate constant in the deactivation kinetics, $K_{d}$, is taken to be independent of the temperature of operation.

9. All reactors use similar types of catalysts, that are identical in functioning and performance (That is, identical values of $K_{d}$ ).

10. For each reactor, there is a maximum number of catalyst loads that can be used over the given time horizon. This number is the same for all reactors.

11. For each reactor, the time required for the maintenance action of shutting down the reactor, replacing the catalyst and restarting operation, is taken to be one month, during which time no production occurs.

12. The availability of labour and equipment in the process is such that in any one month, only one reactor can undergo catalyst replacement.

13. The main reaction is assumed to be of the form:

$$
R \rightarrow Q
$$

where $R$ is the reactant and $Q$ is the desired product. The reaction rate is considered separable from the catalyst activity and is first order with respect to the concentration of the reactant, $R$. That is, the reaction rate equation is of general form:

$$
K_{R} \times c a t_{-} a c t \times c R
$$


where $K_{R}$ is the reaction rate constant, cat_act is the catalyst activity and $c R$ is the concentration of reactant exiting the reactor.

14. The reaction rate constant, $K_{R}$, is taken to exhibit an Arrhenius form of temperature dependence.

15. The flow rate of feed to the process has an upper limit. That is, the sum of feed flow rates to all reactors cannot exceed this limit.

16. The feed flow rate to each reactor has to be specified on a weekly basis.

17. For each reactor, the flow of feed is stopped during the maintenance action of catalyst replacement.

18. The concentration of reactant $R$ in the feed to the process is known and constant.

19. The temperature of operation of each reactor has to be specified on a weekly basis.

20. The temperature of each reactor can be operated only within fixed bounds during catalyst operation and is set to its lower bound during catalyst replacement.

21. The product produced by all reactors is stored continuously as inventory.

22. The weekly product demand is known for the whole time horizon.

23. The amount of product sales from the inventory present has to be specified on a weekly basis.

24. The product sales for each week is less than or equal to the demand in that week.

25. There is a penalty corresponding to the unmet demand in each week. 
26. The costs involved in the process are known and are subject to a known value of annual inflation. These include the sales price of the product, the cost of inventory, the cost of flow and raw material, the cost of catalyst changeover and the penalty for unmet demand.

Given the above assumptions, the optimisation model must determine the following sets of values, which constitute the controls of the MSMIOCP:

(i) The catalyst changeover decision variables, for each month $i$, for reactors $1,2,3$ and 4 , represented by symbols $y 1(i), y 2(i), y 3(i)$ and $y 4(i)$, respectively. For reactor $1, y 1(i)=1$ indicates that a catalyst is in operation and $y 1(i)=0$ indicates that the catalyst is being replaced, during month $i$. An analogous description applies for variables $y 2(i)$, $y 3(i)$ and $y 4(i)$ in reactors 2,3 and 4 , respectively

(ii) The feed flow rate to reactors $1,2,3$ and 4 , during each week, $j$, of each month, $i$, represented by symbols $f f r 1(i, j), f f r 2(i, j), f f r 3(i, j)$ and $f f r 4(i, j)$, respectively

(iii) The amount of product sold, at the end of each week, $j$, of each month, $i$, represented by sales $(i, j)$

(iv) The temperature of operation of reactors 1, 2, 3 and 4, during each week, $j$, of each month, $i$, represented by symbols $T 1(i, j), T 2(i, j)$, $T 3(i, j)$ and $T 4(i, j)$, respectively

In the above list, $j \in\{1,2,3,4\}$ and $i \in\{1,2, \ldots, N M\}$. The catalyst changeover decisions correspond to the binary controls $u$ in equation (4f) while the other decision variables correspond to continuous controls $v$ in equation $(4 \mathrm{~g})$.

The state variables that characterise the MSMIOCP formulation of this industrial process include the following sets of variables: 
(i) The ages of the catalysts in reactors 1, 2, 3 and 4, represented by symbols cat_age1, cat_age 2 , cat_age 3 and cat_age4, respectively

(ii) The activities of the catalysts in reactors 1, 2, 3 and 4, represented by symbols cat_act 1 , cat_act 2 , cat_act 3 and cat_act 4 , respectively

(iii) The concentration of the reactant at the exit of the reactors 1,2,3 and 4 , represented by symbols $c R 1, c R 2, c R 3$ and $c R 4$, respectively

(iv) The product inventory level, inl

(v) The cumulative inventory costs, cum_inc $_{-}$

These state variables are determined by the decision variables' values at any time using a set of ODEs and under the influence of constraints. Next, ODEs of the form of equation (4b), that apply for week $j \in\{1,2,3,4\}$ of month $i \in\{1,2, \ldots, N M\}$ of the process are formulated.

1. In all reactors, the catalyst age varies linearly with time when the catalyst is in operation $(y 1(i), y 2(i), y 3(i), y 4(i)=1)$ but does not increase at times of catalyst replacement $(y 1(i), y 2(i), y 3(i), y 4(i)=0)$. Hence, the differential equations describing the catalyst age in reactors 1, 2, 3 and 4, accounting for both scenarios, are given by equations, (10a), (10b), (10c) and (10d), respectively:

$$
\begin{aligned}
& \frac{d(\text { cat_age } 1)}{d t}=y 1(i) \\
& \frac{d(\text { cat_age } 2)}{d t}=y 2(i) \\
& \frac{d\left(c a t_{-} a g e 3\right)}{d t}=y 3(i) \\
& \frac{d\left(c a t_{-} a g e 4\right)}{d t}=y 4(i)
\end{aligned}
$$


2. In all reactors, the catalyst activity decays on a first order basis during times of catalyst operation $(y 1(i), y 2(i), y 3(i), y 4(i)=1)$ but experiences no change during times of catalyst replacement $(y 1(i), y 2(i)$, $y 3(i), y 4(i)=0)$, as there is no production occurring. Thus, the differential equations for the catalyst activity in reactors 1, 2, 3 and 4, accounting for both scenarios, are given by equations, (11a), (11b), (11c) and (11d), respectively:

$$
\begin{aligned}
& \frac{d\left(c a t_{-} a c t 1\right)}{d t}=y 1(i) \times\left[-K_{d} \times c a t_{-} a c t 1\right] \\
& \frac{d\left(c a t_{-} a c t 2\right)}{d t}=y 2(i) \times\left[-K_{d} \times c a t_{-} a c t 2\right] \\
& \frac{d\left(c a t_{-} a c t 3\right)}{d t}=y 3(i) \times\left[-K_{d} \times c a t_{-} a c t 3\right] \\
& \frac{d\left(c a t_{-} a c t 4\right)}{d t}=y 4(i) \times\left[-K_{d} \times c a t_{-} a c t 4\right]
\end{aligned}
$$

where $K_{d}$ is the catalyst deactivation rate constant.

3. Since all reactors are assumed to be completely stirred, the concentration of reactant exiting each reactor $(c R 1, c R 2, c R 3, c R 4)$ is obtained from the generic mass balance equation of a CSTR during times of catalyst operation $(y 1(i), y 2(i), y 3(i), y 4(i)=1)$. However, during times of catalyst replacement $(y 1(i), y 2(i), y 3(i), y 4(i)=0)$, no reaction occurs and an artificial condition is imposed wherein the reactor is assumed to be filled with fresh, unreacted reactant at the entry concentration $(C R 0)$, to be used by the new catalyst after replacement. The differential equations that account for both scenarios, for reactors 1, 2, 3 and 4 are given by equations, (12a), (12b), (12c) and (12d), respectively:

$$
\begin{aligned}
\frac{d(V \times c R 1)}{d t}= & f f r 1(i, j) \times(C R 0-c R 1) \\
& -y 1(i) \times\left[V \times K 1_{R} \times c a t_{-} a c t 1 \times c R 1\right]
\end{aligned}
$$




$$
\begin{aligned}
\frac{d(V \times c R 2)}{d t}= & f f r 2(i, j) \times(C R 0-c R 2) \\
& -y 2(i) \times\left[V \times K 2_{R} \times c a t_{-} a c t 2 \times c R 2\right] \\
\frac{d(V \times c R 3)}{d t}= & f f r 3(i, j) \times(C R 0-c R 3) \\
& -y 3(i) \times\left[V \times K 3_{R} \times c a t_{-} a c t 3 \times c R 3\right] \\
\frac{d(V \times c R 4)}{d t}= & f f r 4(i, j) \times(C R 0-c R 4) \\
& -y 4(i) \times\left[V \times K 4_{R} \times c a t_{-} a c t 4 \times c R 4\right]
\end{aligned}
$$

Here $V$ is the volume, considered equal for all reactors. $K 1_{R}, K 2_{R}$, $K 3_{R}$ and $K 4_{R}$ are the rate constants for the reactions occurring in reactors 1, 2, 3 and 4, respectively, and they exhibit an Arrhenius form of dependence on the temperature of operation of the respective reactors, of the following form:

$$
\begin{aligned}
& K 1_{R}=A_{R} \times \exp \left(-\frac{E_{a c t}}{R_{g} \times T 1(i, j)}\right) \\
& K 2_{R}=A_{R} \times \exp \left(-\frac{E_{a c t}}{R_{g} \times T 2(i, j)}\right) \\
& K 3_{R}=A_{R} \times \exp \left(-\frac{E_{a c t}}{R_{g} \times T 3(i, j)}\right) \\
& K 4_{R}=A_{R} \times \exp \left(-\frac{E_{a c t}}{R_{g} \times T 4(i, j)}\right)
\end{aligned}
$$

Here $A_{R}$ and $E_{\text {act }}$ are the pre-exponential factor and the activation energy, respectively, of reaction (8) and $R_{g}$ is the universal gas constant.

4. It is assumed that product produced by all reactors is stored as inventory before being sold at the end of the week. During times of catalyst operation $(y 1(i), y 2(i), y 3(i), y 4(i)=1)$ in a reactor, the inventory level increases equivalent to the production rate (volume times 
reaction rate) of that reactor. But during catalyst replacement ( $y 1(i)$, $y 2(i), y 3(i), y 4(i)=0)$ in a reactor, the reactor does not contribute to an increase in inventory level as there is no production occurring. So, the differential equation describing the inventory level $(\mathrm{inl})$, that takes into account production from all reactors, while considering scenarios of catalyst operation as well as replacement, is given by:

$$
\begin{aligned}
& \frac{d(i n l)}{d t}=\quad y 1(i) \times\left[V \times K 1_{R} \times c a t \_a c t 1 \times c R 1\right] \\
& +y 2(i) \times\left[V \times K 2_{R} \times c a t_{-} a c t 2 \times c R 2\right] \\
& +y 3(i) \times\left[V \times K 3_{R} \times c a t_{-} a c t 3 \times c R 3\right] \\
& +y 4(i) \times\left[V \times K 4_{R} \times c a t_{-} a c t 4 \times c R 4\right]
\end{aligned}
$$

5. Finally, the increase in the cumulative inventory cost (cum_inc) at any time depends on the inventory level at that time and the Inventory Cost Factor (icf) (adjusted for inflation), which stipulates the cost per unit product per unit time:

$$
\frac{d\left(c u m_{-} i n c\right)}{d t}=i n l \times i c f
$$

The $i c f$ at any time is given by the following equation:

$$
i c f=b_{a s e} i c f \times(1+\text { inflation })^{\lfloor i / 12\rfloor}
$$

where base_icf is the inventory cost factor before inflation, inflation is the annual inflation rate and $\lfloor\cdot\rfloor$ is the greatest integer function.

The set of ODEs are solved repeatedly over a weekly time span, which corresponds to one stage of the MSMIOCP. In order to solve these ODEs, for each stage, suitable initial conditions have to be provided. The initial conditions for week 1 of month 1 are assumed to be known and are of the form of equation (4c). The initial conditions for the other stages are obtained 
using junction conditions between two successive stages of the process, of the form of equation $(4 \mathrm{~d})$.

The initial conditions corresponding to week 1 of month 1, represented as init_var $(1,1)$ for variable var, are as follows:

1. In all reactors, a new catalyst is used at the beginning of the process and so the initial catalyst age in all reactors is set to zero:

$$
\begin{aligned}
& \text { init_cat_age } 1(1,1)=0 \\
& \text { init_cat_age } 2(1,1)=0 \\
& \text { init_cat_age } 3(1,1)=0 \\
& \text { init_cat_age } 4(1,1)=0
\end{aligned}
$$

2. Since, in all reactors, a new catalyst is used at the beginning of the process, the initial catalyst activity for the catalysts in all reactors is set to that of a fresh catalyst (start_cat_act):

$$
\begin{aligned}
& \text { init_cat_act } 1(1,1)=\text { start_cat_act } \\
& \text { init_cat_act } 2(1,1)=\text { start_cat_act } \\
& \text { init_cat_act } 3(1,1)=\text { start_cat_act } \\
& \text { init_cat_act } 4(1,1)=\text { start_cat_act }
\end{aligned}
$$

3. At the start of the process, all reactors are filled with the reactant $R$ at its entry concentration $C R 0$. Hence, the initial exit concentration in all reactors is given by:

$$
\text { init_cR1 }(1,1)=C R 0
$$




$$
\begin{aligned}
& \text { init_cR2 }(1,1)=C R 0 \\
& \text { init_cR3 }(1,1)=C R 0 \\
& \text { init_cR4 }(1,1)=C R 0
\end{aligned}
$$

4. There is no inventory at the beginning of the process, and so:

$$
\text { init_inl }(1,1)=0
$$

5. There is no inventory at the start of the process and so the initial cumulative inventory cost is nil at that time:

$$
\text { init_cum_inc }(1,1)=0
$$

The junction conditions are described next. For all reactors, the junction conditions differ depending on whether the catalyst is in operation $(y 1(i)$, $y 2(i), y 3(i), y 4(i)=1)$ or is being replaced ( $y 1(i), y 2(i), y 3(i), y 4(i)=0)$ during that month. In the following text, the expressions init_var $(i, j)$ and end_var $(i, j)$ indicate the initial and end conditions, respectively for the variable var, for week $j$ of month $i$ :

1. During months of catalyst operation $(y 1(i), y 2(i), y 3(i), y 4(i)=1)$, in all reactors, the initial catalyst age for a week corresponds to the catalyst age at the end of the previous week. But during months of catalyst replacement $(y 1(i), y 2(i), y 3(i), y 4(i)=0)$, the catalyst age has to be set to zero, the age of a new catalyst. The junction conditions that describe both scenarios for all reactors, are as follows.

$$
\begin{aligned}
& \text { init_cat_age } 1(i, j+1)=\text { end_cat_age } 1(i, j) \\
& \text { init_cat_age } 2(i, j+1)=\text { end_cat_age } 2(i, j) \\
& \text { init_cat_age } 3(i, j+1)=\text { end_cat_age } 3(i, j)
\end{aligned}
$$




$$
\begin{array}{r}
\text { init_cat_age }(i, j+1)=\text { end_cat_age } 4(i, j) \\
j=1,2,3 \quad i=1,2, \ldots, N M \\
\text { init_cat_age }(i, 1)=y 1(i) \times \text { end_cat_age } 1(i-1,4) \\
\text { init_cat_age } 2(i, 1)=y 2(i) \times \text { end_cat_age } 2(i-1,4) \\
\text { init_cat_age } 3(i, 1)=y 3(i) \times \text { end_cat_age } 3(i-1,4) \\
\text { init_cat_age }(i, 1)=y 4(i) \times \text { end_cat_age } 4(i-1,4) \\
i=2,3, \ldots, N M
\end{array}
$$

2. During months of catalyst operation $(y 1(i), y 2(i), y 3(i), y 4(i)=1)$, in all reactors, the initial catalyst activity for the week corresponds to the catalyst activity at the end of the previous week. However, during months of catalyst replacement $(y 1(i), y 2(i), y 3(i), y 4(i)=0)$, in all reactors, the catalyst activity has to be reset to the activity corresponding to that of a fresh catalyst, which remains the same throughout the duration of month $i$. The junction conditions that describe both scenarios for all reactors is given as follows.

$$
\begin{array}{r}
\text { init_cat_act } 1(i, j+1)=\text { end_cat_act } 1(i, j) \\
\text { init_cat_act } 2(i, j+1)=\text { end_cat_act } 2(i, j) \\
\text { init_cat_act } 3(i, j+1)=\text { end_cat_act } 3(i, j) \\
\text { init_cat_act } 4(i, j+1)=\text { end_cat_act } 4(i, j) \\
j=1,2,3 \quad i=1,2, \ldots, N M \\
\text { init_cat_act } 1(i, 1)=[y 1(i) \times \text { end_cat_act } 1(i-1,4)] \\
+[(1-y 1(i)) \times \text { start_cat_act }]
\end{array}
$$




$$
\begin{aligned}
\text { init_cat_act } 2(i, 1)= & {[y 2(i) \times \text { end_cat_act } 2(i-1,4)] } \\
& +[(1-y 2(i)) \times \text { start_cat_act }] \\
\text { init_cat_act } 3(i, 1)= & {[y 3(i) \times \text { end_cat_act } 3(i-1,4)] } \\
& +[(1-y 3(i)) \times \text { start_cat_act }] \\
\text { init_cat_act }(i, 1)= & {[y 4(i) \times \text { end_cat_act } 4(i-1,4)] } \\
& +[(1-y 4(i)) \times \text { start_cat_act }] \\
& i=2,3, \ldots, N M
\end{aligned}
$$

3. During months of catalyst operation $(y 1(i), y 2(i), y 3(i), y 4(i)=1)$, in all reactors, the exit concentration for the beginning of a week corresponds to the exit concentration at the end of the previous week. And when the catalyst is being replaced $(y 1(i), y 2(i), y 3(i), y 4(i)=0)$ in a reactor, an artificial condition is imposed wherein the reactor is filled with reactant at entry concentration $C R 0$, ready to be used by the fresh catalyst at the beginning of the next month. The junction conditions that describe both scenarios for all reactors is given as follows.

$$
\begin{aligned}
& \text { init_cR1 }(i, j+1)=\text { end_cR } 1(i, j) \\
& \text { init_cR2 }(i, j+1)=\text { end_cR }_{-}(i, j) \\
& \text { init_cR3 }(i, j+1)=\text { end_cR }_{-}(i, j) \\
& \text { init_cR4 }(i, j+1)=\text { end_c }_{-} 4(i, j) \\
& j=1,2,3 \quad i=1,2, \ldots, N M \\
& \text { init_cR } 1(i, 1)=\left[y 1(i) \times e n d_{-} c R 1(i-1,4)\right]+[(1-y 1(i)) \times C R 0] \\
& \text { init_cR2 }(i, 1)=\left[y 2(i) \times e n d_{-} c R 2(i-1,4)\right]+[(1-y 2(i)) \times C R 0]
\end{aligned}
$$




$$
\begin{aligned}
& \text { init_cR3(i, })=\left[y 3(i) \times e n d_{-} c R 3(i-1,4)\right]+[(1-y 3(i)) \times C R 0] \\
& \text { init_cR4 }(i, 1)=\left[y 4(i) \times e n d_{-} c R 4(i-1,4)\right]+[(1-y 4(i)) \times C R 0]
\end{aligned}
$$

$$
i=2,3, \ldots, N M
$$

4. At the end of a week, an amount, $\operatorname{sales}(i, j)$ of the stored product is sold. Thus, the initial inventory level for the week corresponds to the inventory present after the sales at the end of the previous week. The following junction conditions apply during months of catalyst operation as well as catalyst replacement, as the sales do not cease at any time:

$$
\begin{gathered}
\text { init_inl }(i, j+1)=\text { end_inl }(i, j)-\operatorname{sales}(i, j) \\
j=1,2,3 \quad i=1,2, \ldots, N M \\
\text { init_inl }(i, 1)=\text { end_inl }(i-1,4)-\operatorname{sales}(i-1,4) \\
i=2,3, \ldots, N M
\end{gathered}
$$

5. The inventory cost accumulated until the beginning of a week is equal to the value of the inventory cost accumulated until the end of the previous week. So the following junction conditions apply regardless of whether the catalyst is being used or replaced:

$$
\begin{gathered}
\text { init_cum_inc }(i, j+1)=\text { end_cum_inc }(i, j) \\
j=1,2,3 \quad i=1,2, \ldots, N M \\
\text { init_cum_inc }(i, 1)=\text { end_cum_inc }(i-1,4) \\
i=2,3, \ldots, N M
\end{gathered}
$$

The initial conditions (17) - (21) and junction conditions (22) - (26) enable a solution for the ODEs for all stages and thereby obtain the values of the state variables. However, in order to ensure the feasibility of the obtained 
state variables values, these ODEs have to be solved in tandem with fulfilling the constraints of the process.

The constraints, of the form of equation (4e), that apply to this industrial process for week $j \in\{1,2,3,4\}$ of month $i \in\{1,2, \ldots, N M\}$ are as follows:

1. In the context of solving the MSMIOCP as a standard MSOCP using a feasible path approach, the catalyst changeover decision variables for all reactors $(y 1(i), y 2(i), y 3(i), y 4(i))$, for a month $i$, are considered continuous variables that vary between 0 and 1 , and so the following bounds are imposed:

$$
\begin{aligned}
& 0 \leq y 1(i) \leq 1 \\
& 0 \leq y 2(i) \leq 1 \\
& 0 \leq y 3(i) \leq 1 \\
& 0 \leq y 4(i) \leq 1
\end{aligned}
$$

2. The net flow rate of feed to the process has an upper limit $(F U p)$. That is, the sum of the flow rates of feeds to all reactors has to remain within this limit and so, the following bounds are imposed, for each week:

$$
0 \leq f f r 1(i, j)+f f r 2(i, j)+f f r 3(i, j)+f f r 4(i, j) \leq F U p
$$

3. The sales in each week are assumed to be less than or equal to the demand for the product in that week $(\operatorname{demand}(i, j))$. Hence, the following bounds on the sales at the end of each week are imposed:

$$
0 \leq \operatorname{sales}(i, j) \leq \operatorname{demand}(i, j)
$$

4. The temperature of each reactor operates between known, fixed lower 
and upper bounds, TLo and TUp, respectively. Hence, the following bounds are set on the weekly temperature of operation of each reactor:

$$
\begin{aligned}
& T L o \leq T 1(i, j) \leq T U p \\
& T L o \leq T 2(i, j) \leq T U p \\
& T L o \leq T 3(i, j) \leq T U p \\
& T L o \leq T 4(i, j) \leq T U p
\end{aligned}
$$

5. When a catalyst is being replaced in a reactor $(y 1(i), y 2(i), y 3(i)$, $y 4(i)=0$ ), the flow of raw material to that reactor stops. The following constraints ensure that the weekly feed flow rate to each reactor remains below the upper bound during times of catalyst operation $(y 1(i), y 2(i)$, $y 3(i), y 4(i)=1)$ and drops to zero when there is catalyst replacement (y1(i), $y 2(i), y 3(i), y 4(i)=0)$ :

$$
\begin{aligned}
& f f r 1(i, j)-[F U p \times y 1(i)] \leq 0 \\
& f f r 2(i, j)-[F U p \times y 2(i)] \leq 0 \\
& f f r 3(i, j)-[F U p \times y 3(i)] \leq 0 \\
& f f r 4(i, j)-[F U p \times y 4(i)] \leq 0
\end{aligned}
$$

6. When a catalyst is being replaced in a reactor $(y 1(i), y 2(i), y 3(i)$, $y 4(i)=0)$, the temperature of the reactor is required to drop to its lower bound. This condition is imposed using the following constraints which ensure that the temperature of each reactor, for each week, remains between its bounds during times of catalyst operation (y1(i), $y 2(i), y 3(i), y 4(i)=1$ ) but drops to the lower bound when there is 
catalyst replacement $(y 1(i), y 2(i), y 3(i), y 4(i)=0)$ :

$$
\begin{aligned}
& T L o \leq T 1(i, j) \leq[(T U p-T L o) \times y 1(i)]+T L o \\
& T L o \leq T 2(i, j) \leq[(T U p-T L o) \times y 2(i)]+T L o \\
& T L o \leq T 3(i, j) \leq[(T U p-T L o) \times y 3(i)]+T L o \\
& T L o \leq T 4(i, j) \leq[(T U p-T L o) \times y 4(i)]+T L o
\end{aligned}
$$

7. For each reactor, there is a maximum number of catalyst replacements that can occur. In this case study, it is assumed that this maximum number is the same for all reactors (designated as $n$ ). The limit on the maximum number of catalyst changeovers allowed for each reactor is imposed using the following set of constraints:

$$
\begin{aligned}
& \sum_{i=1}^{N M} y 1(i) \geq N M-n \\
& \sum_{i=1}^{N M} y 2(i) \geq N M-n \\
& \sum_{i=1}^{N M} y 3(i) \geq N M-n \\
& \sum_{i=1}^{N M} y 4(i) \geq N M-n
\end{aligned}
$$

8. The availability of equipment and labour in the process is such that only one reactor can undergo catalyst replacement $(y 1(i), y 2(i), y 3(i)$, $y 4(i)=0)$ during any month. Mathematically, this means that among the catalyst changeover controls for all reactors $(y 1(i), y 2(i), y 3(i)$, $y 4(i)$ ), at most one control can take a value of 0 (or at least 3 controls should have values of 1 ) during any month, $i$. This condition of 
non-simultaneous catalyst replacement is enforced using the following constraint:

$$
y 1(i)+y 2(i)+y 3(i)+y 4(i) \geq 3
$$

9. The catalysts in all reactors undergo deactivation over time and have to be replaced before crossing a certain maximum age. Since all reactors use catalysts that are identical in functioning and performance, a common for all maximum catalyst age, designated as max_cat_age, is used. As the decision on whether to replace a catalyst or not is made on a monthly basis, it is sufficient to ensure that the catalyst age in each reactor does not cross this limit at the end of each month, $i$ :

$$
\begin{aligned}
& \text { end_cat_age } 1(i, 4) \leq \text { max_cat_age } \\
& \text { end_cat_age } 2(i, 4) \leq \text { max_cat_age } \\
& \text { end_cat_age } 3(i, 4) \leq \text { max_cat_age } \\
& \text { end_cat_age } 4(i, 4) \leq \text { max_cat_age }
\end{aligned}
$$

10. In order to ensure that more product than available is not sold, the inventory level at the end of each week should be greater than the sales for the week. This is imposed using the following constraint:

$$
\operatorname{end} \operatorname{inl}(i, j)-\operatorname{sales}(i, j) \geq 0
$$

The aim is to maximise the profits or minimise the costs of the process under the influence of these ODEs, initial conditions, junction conditions and constraints. The net costs of the process are represented by the objective function of this MSMIOCP, of the form of equation (4a), and comprises of the following elements:

1. The Gross Revenue from Sales $(G R S)$ 
This term represents the revenue for the process from the net sales of the product chemical over the whole time horizon:

$$
G R S=\sum_{i=1}^{N M} \sum_{j=1}^{4} p s p(i, j) \times \operatorname{sales}(i, j)
$$

where $\operatorname{psp}(i, j)$ is the sales price per unit product for week $j$ of month $i$, adjusted for inflation at that time:

$$
p s p(i, j)=b_{\text {bse_psp }} \times(1+\text { inflation })^{\lfloor i / 12\rfloor}
$$

where base_psp is the unit product sales price before inflation.

2. The Total Inventory Costs (TIC)

This term represents the net storage costs for the product over the whole time horizon and is obtained from the solution of the ODEs for the state variable cum_inc at the end of the final week of the process:

$$
T I C=\text { end_cum_inc }(N M, 4)
$$

3. The Total Costs of Catalyst Changeovers $(T C C C)$

The total expenditure for the catalyst changeover operations is given by the sum of the catalyst changeover costs for all 4 reactors. Since these costs are incurred only during months of catalyst replacement $(y 1(i)$, $y 2(i), y 3(i), y 4(i)=0), T C C C$ is obtained in the following manner:

$$
\begin{aligned}
T C C C= & \sum_{i=1}^{N M} \operatorname{crc}(i) \times[1-y 1(i)]+\sum_{i=1}^{N M} \operatorname{crc}(i) \times[1-y 2(i)] \\
& +\sum_{i=1}^{N M} \operatorname{crc}(i) \times[1-y 3(i)]+\sum_{i=1}^{N M} \operatorname{crc}(i) \times[1-y 4(i)]
\end{aligned}
$$

It is highlighted that the terms within the summations remain non- 
zero only during the times of catalyst replacement $(y 1(i), y 2(i), y 3(i)$, $y 4(i)=0)$ and only these terms contribute to the total costs. Here $\operatorname{crc}(i)$ is the cost of the catalyst replacement operation, considered the same for all reactors, for month $i$, adjusted for inflation at that time:

$$
\operatorname{crc}(i)=\text { base_crc } \times(1+\text { inflation })^{\lfloor i / 12\rfloor}
$$

where base_crc is the cost of a catalyst changeover operation before inflation.

4. The Net Penalty for Unmet Demand (NPUD)

The unmet demand in each week (unmet_demand $(i, j)$ ) is the quantity of product by which the sales falls short of the demand in that week:

$$
\begin{gathered}
\text { unmet_demand }(i, j)=\operatorname{demand}(i, j)-\operatorname{sales}(i, j) \\
j=1,2,3,4 \quad i=1,2, \ldots, N M
\end{gathered}
$$

There is a penalty associated with this unmet demand and the net penalty costs over the entire time horizon is given by:

$$
N P U D=\sum_{i=1}^{N M} \sum_{j=1}^{4} \operatorname{pen}(i, j) \times \text { unmet_demand }(i, j)
$$

where $\operatorname{pen}(i, j)$ is the penalty per unit product for week $j$ of month $i$, adjusted for inflation at that time:

$$
\operatorname{pen}(i, j)=\text { base }_{-} \text {pen } \times(1+\text { inflation })^{\lfloor i / 12\rfloor}
$$

where base_pen is the penalty per unit product before inflation.

5. The Total Flow Costs $(T F C)$

This term represents the net expenditure on the feed of raw material 
to all reactors in the process and is given by:

$T F C=\sum_{i=1}^{N M} \sum_{j=1}^{4} \operatorname{cof}(i, j) \times[f f r 1(i, j)+f f r 2(i, j)+f f r 3(i, j)+f f r 4(i, j)]$

where $\operatorname{cof}(i, j)$ is the cost of raw material per unit volume per week for week $j$ of month $i$, adjusted for inflation at that time:

$$
\operatorname{cof}(i, j)=\text { base_cof } \times(1+\text { inflation })^{\lfloor i / 12\rfloor}
$$

where base_cof is the cost of raw material per unit volume per week before inflation.

If the Net Costs are represented by $N C$, the objective function for this optimisation problem takes the form:

$$
\min N C=-G R S+T I C+T C C C+N P U D+T F C
$$

The essential elements of the problem formulation have now been described in detail. The aim is to make the appropriate decisions in order to minimise the net costs (or maximise the profit) of the industrial process, when subject to the ODEs, initial and junction conditions and the constraints.

Next, the solution implementation details are presented after which the results obtained are discussed. The set of parameters used for this problem are given in Table 1, which are mostly similar to those used in Adloor et al. (2020), except here the volumes of the 4 reactors used add up to that of the single reactor in the latter work. A 3-year time horizon is considered here as well and the details of the problem size under this formulation for this horizon length is given in Table 2 . 


\subsection{Implementation details}

The implementation as a standard MSOCP using the feasible path approach was performed in Python ${ }^{\mathrm{TM}}$ 3.7.1 under PyCharm 2019.3.3 (Community Edition). The code was written using CasADi, an open source software that enables a symbolic framework for numerical optimisation (Andersson, 2013). The elements of the problem, as given in Section 4.1, were defined as symbolic expressions using CasADi v3.4.5. The Automatic Differentiation (AD) feature of CasADi enabled constructions of symbolic expressions of the derivatives of all predefined functions, thereby maintaining differentiability to an arbitrary order. This allowed for an efficient calculation of gradients, that did not suffer from round-off and truncation errors, unlike gradient calculation using finite differences.

For the choice of parameters used in this article, the weight term in the objective function as per the penalty term homotopy technique presented in equation (6) in Section 3 is increased as per the arithmetic progression in equation (48):

Table 1: List of parameters

\begin{tabular}{|c|c|}
\hline Parameter Symbol & Value \\
\hline \hline$A_{R}$ & $885(1 /$ day $)$ \\
\hline base_cof & $\$ 210 /$ week \\
\hline base_crc & $\$ 25 \times 10^{5}$ \\
\hline base_icf & $\$ 0.01 /(\mathrm{kmol}$ day $)$ \\
\hline base_pen & $\$ 1250 / \mathrm{kmol}$ \\
\hline base_psp & $\$ 1000 / \mathrm{kmol}^{-}$ \\
\hline$C R 0$ & $1 \mathrm{kmol} / \mathrm{m}^{3}$ \\
\hline
\end{tabular}


Table 1: List of parameters

\begin{tabular}{|c|c|}
\hline Parameter Symbol & Value \\
\hline \hline \multirow{2}{*}{ demand } & 1st quarter of year: $8000 \mathrm{kmol} /$ week \\
\cline { 2 - 2 } & 2nd quarter of year: $7200 \mathrm{kmol} /$ week \\
\cline { 2 - 2 } & 3rd quarter of year: $3300 \mathrm{kmol} /$ week \\
\cline { 2 - 2 }$E_{\text {act }}$ & 4th quarter of year: $4500 \mathrm{kmol} /$ week \\
\hline FUp & $30,000 \mathrm{~J} /$ gmol \\
\hline inflation & $9600 \mathrm{~m}^{3} /$ day \\
\hline Kd & $5 \%$ \\
\hline max_cat_age & $0.0024(1 /$ day $)$ \\
\hline$n$ & 504 days $(=1.5$ years $)$ \\
\hline$N M$ & 5 \\
\hline$R_{g}$ & 36 months $(=3$ years $)$ \\
\hline start_cat_act & $8.314 \mathrm{~J} /(\mathrm{gmol} . \mathrm{K})$ \\
\hline$T L o$ & 1 \\
\hline$T U p$ & $400 \mathrm{~K}$ \\
\hline$V$ & $1000 \mathrm{~K}$ \\
\hline & $12.5 \mathrm{~m}{ }^{3}$ \\
\hline
\end{tabular}

$$
\begin{gathered}
M_{k+1}=\left(2 \times M_{k}\right)+\left(5 \times 10^{7}\right) \\
M_{1}=0 \\
k=1,2,3 \ldots
\end{gathered}
$$

CasADi plug-ins to the open source SUNDIALS suite (Hindmarsh et al., 
Table 2: Problem size specifications

\begin{tabular}{|c|c|c|}
\hline \multicolumn{2}{|c|}{ Property } & Size \\
\hline \hline Ordinary & Differential Equations & 2016 \\
\hline \hline \multirow{4}{*}{ Decision variables } & Catalyst changeover actions & 144 \\
\cline { 2 - 3 } & Feed flow rate & 576 \\
\cline { 2 - 3 } & Sales & 144 \\
\cline { 2 - 3 } & Temperature & 576 \\
\cline { 2 - 3 } & Total & 1440 \\
\hline \hline \multirow{7}{*}{ Constraints } & Constraints (27) & 288 \\
\cline { 2 - 3 } & Constraints (28) & 288 \\
\cline { 2 - 3 } & Constraints (29) & 288 \\
\cline { 2 - 3 } & Constraints (30) & 1152 \\
\cline { 2 - 3 } & Constraints (31) & 576 \\
\cline { 2 - 3 } & Constraints (32) & 1152 \\
\cline { 2 - 3 } & Constraint (33) & 4 \\
\cline { 2 - 3 } & Constraint (34) & 36 \\
\cline { 2 - 3 } & Constraints (35) & 144 \\
\cline { 2 - 3 } & Constraints (36) & 144 \\
\cline { 2 - 3 } & & 4072 \\
\hline
\end{tabular}

2005) and IPOPT by COIN-OR (Wächter and Biegler, 2006) were used for the integration of ODEs and optimisation, respectively. The IDAS solver of SUNDIALS was used for the integration of the ODEs with the following termination criteria: an absolute tolerance of $10^{-6}$ and a relative tolerance of $10^{-6}$. The optimisation by IPOPT had, respectively, the following termination and 'acceptable' termination criteria: $10^{-4}$ and $10^{-4}$ for the optimality error, 1 and $10^{6}$ for the dual infeasibility, $10^{-4}$ and $10^{-2}$ for the constraint violation, and $10^{-4}$ and $10^{-2}$ for the complementarity. The 'acceptable' num- 
ber of iterations was set at 15 .

The implementation was performed on a $3.2 \mathrm{GHz}$ Intel Core i5, 16 GB RAM, Windows machine running on Microsoft Windows 10 Enterprise. Since the problem is non-convex, multiple runs were performed with different random initial guesses for the decision variables. The random initial guesses were generated using Latin Hypercube Sampling (McKay et al., 1979), obtained in Python using the lhs method of the pyDOE module (version 0.3.8). Test runs using the multiprocessing module in Python, to parallelise a loop of multiple start points, executed slower than when the runs were done serially. So 50 runs were executed in a serial manner.

In the next section, the results obtained using the proposed solution methodology and implementation procedure are discussed. Statistics describing the essential solution features for the 50 runs are provided in the form of tables and figures of the trends of the decision and state variables over the time horizon for the most profitable run are examined.

\subsection{Results and discussions}

As in Adloor et al. (2020), this methodology produced high quality solutions. Regardless of the initial guesses used, each of the 50 runs successfully converged to a local optimum within the specified optimality tolerance, thereby indicating the robustness of the procedure. The nonlinear differential equations were solved to a high accuracy using state-of-the-art integrators, without any linear approximation techniques, thus underscoring the reliability of the obtained solutions. The penalty term homotopy technique was successful, not only in obtaining binary values for the catalyst changeover controls in all reactors but also in enforcing the condition of non-simultaneous catalyst replacement, without the use of mixed-integer programming methods, and this underlines the efficiency of the methodology. 
Table 3: Solution statistics over the 50 multi-start runs

\begin{tabular}{|c||c|c|c|}
\hline Property & Maximum & Minimum & Mean/Mode \\
\hline \hline Profit (Million \$) & 435.595 & 378.817 & 411.855 \\
\hline CPU time (seconds) & 330269 & 174663 & 238882 \\
\hline Number of Major Iterations & 4 & 2 & 3 \\
\hline
\end{tabular}

Statistics regarding the solutions of the set of 50 runs are given in Tables $3-5$. Table 3 provides details of the ranges of the profits, computation times and number of major iterations involved. As can be seen, a minimum of 2 and a maximum of 4 major iterations are needed to obtain binary values for the catalyst changeover controls, with the mode being 3 . The ranges of the number of IPOPT iterations needed within each major iteration are given in Table 4 . Table 5 presents statistics regarding the catalyst ages and the number of catalyst replacements, which are largely similar for all reactors. It is interesting to note that the mean catalyst age for all reactors is about half the maximum age of 504 days and in no run, for any reactor, is a catalyst recommended to be used up to that maximum age. It is also the case that among all runs, for all reactors, only a maximum of 4 catalyst replacements and not all of the available 5 are used, with the mode being 3 .

The trends of the decision and state variables over the time horizon for the most profitable solution among the set of 50 runs are given in Figures 4 - 12. The trends for each reactor are very similar to a related case study in Adloor et al. (2020) for a single reactor.

Figure 4 illustrates the variation of the monthly catalyst changeover controls over the whole time horizon for all 4 reactors. In this case, two major iterations are needed to force the catalyst changeover controls for all reactors 
Table 4: Statistics for each major iteration. The column titled 'Runs' indicates the number of runs out of 50 that progressed until that major iteration. The columns titled 'Max', 'Min' and 'Mean' indicate the maximum, minimum and mean number of IPOPT iterations within that major iteration, respectively.

\begin{tabular}{|c||c|c|c|c|}
\hline Major Iteration & Runs & Max & Min & Mean \\
\hline \hline Major Iteration 1 & 50 & 400 & 206 & 331 \\
\hline Major Iteration 2 & 50 & 200 & 79 & 121 \\
\hline Major Iteration 3 & 44 & 200 & 52 & 76 \\
\hline Major Iteration 4 & 2 & 63 & 58 & 60.5 \\
\hline
\end{tabular}

Table 5: Statistics regarding the catalyst replacements in each reactor

\begin{tabular}{|l||c|c|c|c|c|c|}
\hline \multirow{2}{*}{ Reactor } & \multicolumn{2}{|c|}{ Catalyst age (Days) } & \multicolumn{2}{c|}{ Number of catalyst replacements } \\
\cline { 2 - 7 } & Max & Min & Mean & Max & Min & Mode \\
\hline \hline Reactor 1 & 476 & 112 & 243.2 & 4 & 2 & 3 \\
\hline Reactor 2 & 476 & 112 & 252 & 4 & 2 & 3 \\
\hline Reactor 3 & 476 & 112 & 246 & 4 & 2 & 3 \\
\hline Reactor 4 & 476 & 112 & 234.9 & 4 & 2 & 3 \\
\hline
\end{tabular}

to take integer values. The numerical values of all these controls across both major iterations can be found in Table A.7. From the graph and table, it is seen that the catalyst changeover control for Reactors 1, 2 and 3 (that is, $y 1, y 2$ and $y 3)$ take values of 0 on three occasions while that for Reactor 4 (y4) takes a value of 0 on four occasions. By this, the model indicates that it is optimal for the process to replace the catalyst three times in Reactors 1, 2 and 3 and four times in Reactor 4, during those months corresponding to when their respective catalyst changeover controls become 0 . It is highlighted 
that the months when catalyst replacements occur for the four reactors do not overlap, thereby fulfilling constraint (34) for non-simultaneous catalyst replacement. The other graphs presented are those obtained as solutions of the second major iteration.

The profiles of the feed flow rates to each reactor $(f f r 1, f f r 2, f f r 3$, $f f r 4)$ and the total feed flow rate to the process $(f f r 1+f f r 2+f f r 3+f f r 4)$ over the whole time horizon, are shown in Figures 5 and 6, respectively. The trends of the feed flow rates to all reactors in Figure 5 are related to the corresponding profiles of the temperature of operation $(T 1, T 2, T 3, T 4)$, shown in Figure 8, and the reactant exit concentrations from the reactors $(c R 1, c R 2, c R 3, c R 4)$, shown in Figure 11. An explanation of the trends of all these variables is provided as follows:

- In all reactors, during times of catalyst operation, the feed flow rate is decreased at a rate matching that of the reactor's catalyst deactivation and the temperature of the reactor is maintained at its upper bound. Such an operation causes the reactant exit concentration to maintain a constant value. This operational policy is consistent with the work of Szépe and Levenspiel (1968) for continuous reactors on the lab scale. That paper predicted similar operational strategies when the main reaction is more sensitive to temperature than the catalyst deactivation and the latter is independent of the species' concentration, as is the consideration in this case study.

- During times of catalyst replacement in all reactors, the feed flow rate is set to zero, the temperature of operation is set to its lower bound (TLo) and the reactant exit concentration is set to its entry value $(C R 0)$, as per constraints (31) and (32) and junction conditions (24), respectively.

- In all reactors, during the operation of the last catalyst, a sharp drop in the flow rate occurs that causes a corresponding effect in the exit 
(a) Reactor 1

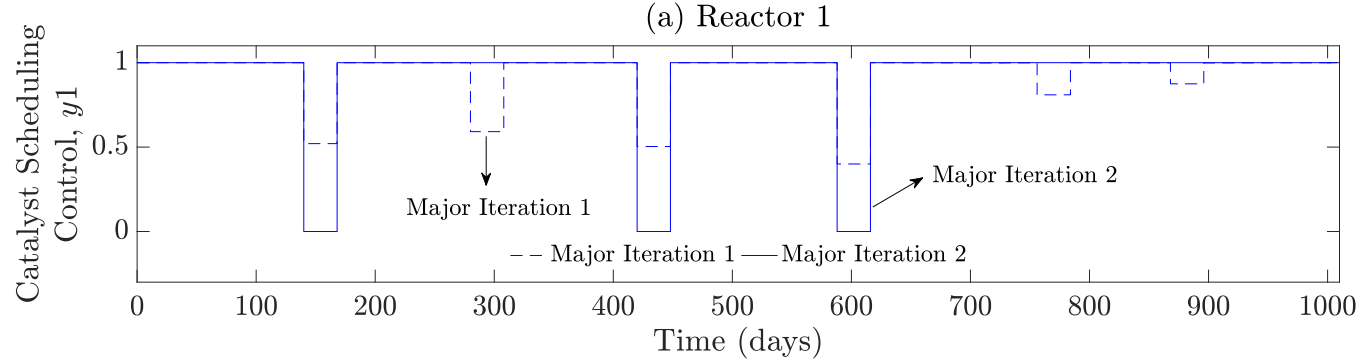

(b) Reactor 2

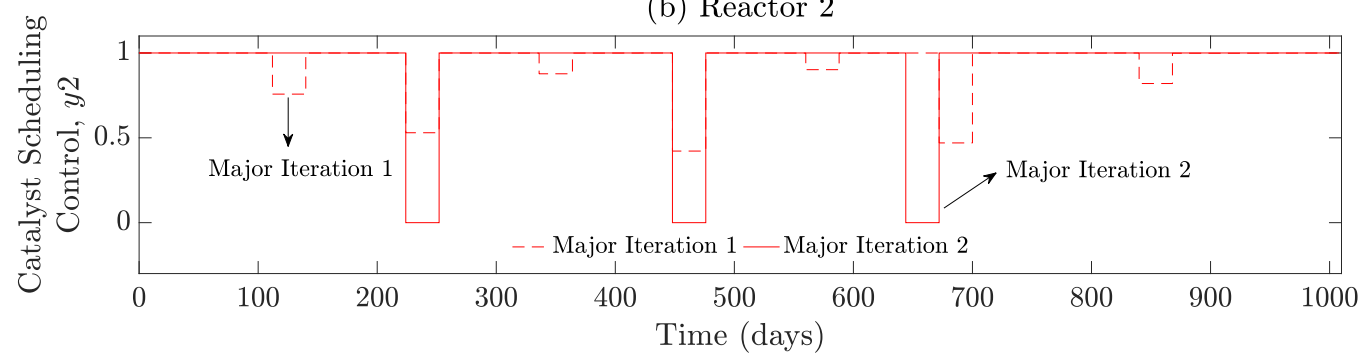

(c) Reactor 3

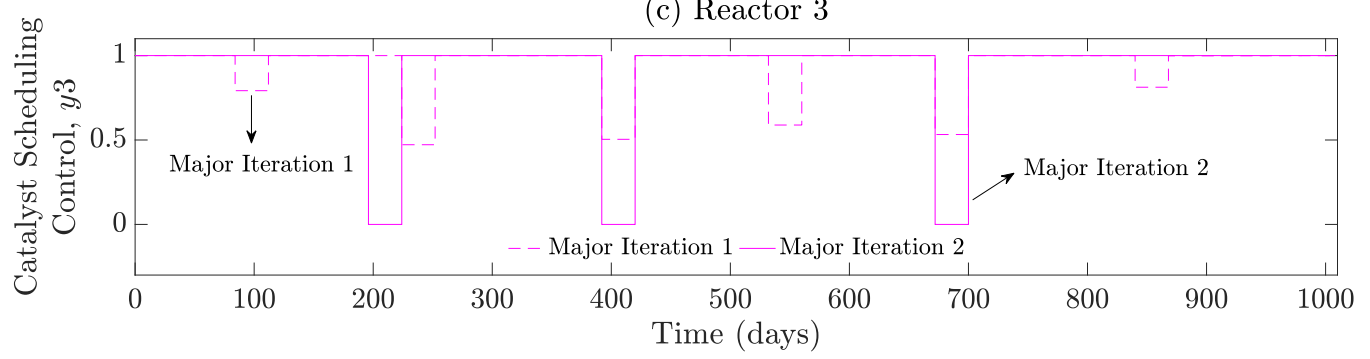

(d) Reactor 4

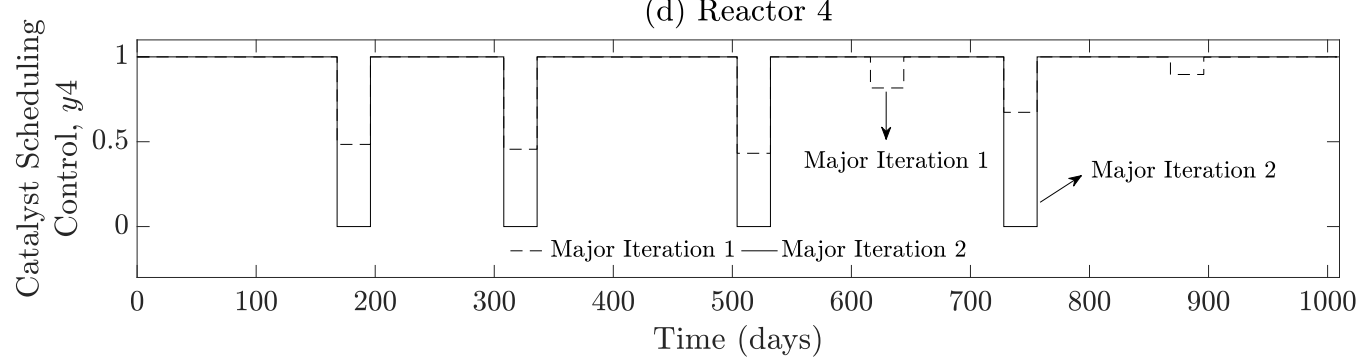

Figure 4: The variation of the catalyst changeover controls over the time horizon in (a) Reactor 1 (b) Reactor 2 (c) Reactor 3 (d) Reactor 4. 
concentration. This occurs to bring the production rate to a value that exactly fulfils the demand for the remainder of the time horizon and thereby lower the costs of flow and raw material to a minimum.

In Figure 6, the profile of the net feed flow rate to the process over the time horizon, does not follow a regular trend, due to the variations in the feed flow rates to each reactor, that operate independently of each other. It is noted from the graph that the net flow rate does not reach its upper bound $(F U p)$ at any point throughout the time horizon, indicating that the optimal policy does not require the maximum feed to the process.

Figure 7 shows a comparison of the optimal quantity of product sales with the corresponding product demand and unmet demand over the whole time horizon. It is seen that a considerable amount of unmet demand exists during the first year of the process, but is nil for the remainder years. This is because the model takes into account the increase in sales price due to annual inflation and prefers to sell less during the first year and more in the later years in order to accumulate greater profit. It is highlighted that the use of parallel reactor lines and the condition that only one reactor can undergo catalyst replacement at any time, enables the sales to occur continuously throughout the time horizon. Taking inflation into account, the model operates the sales in an efficient manner such that the inventory level (Figure 12) is adjusted to balance the trade-offs between storing a sufficient quantity of product to meet seasonal demand and high storage costs.

The variation of the catalyst age over the time horizon for the 4 reactors are shown in Figure 9. The trends of a linear increase with time during catalyst operation and a constant value of 0 during catalyst replacement follow directly from differential equations (10) and junction conditions (22). An analogous graph for catalyst activities in the 4 reactors are shown in Figure 10. In this figure, the trends of an exponential decrease during catalyst 
(a) Reactor 1

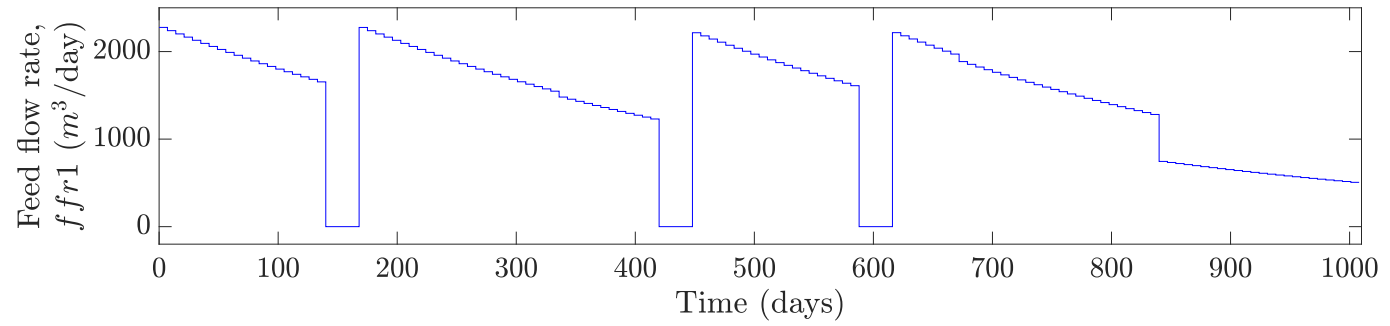

(b) Reactor 2

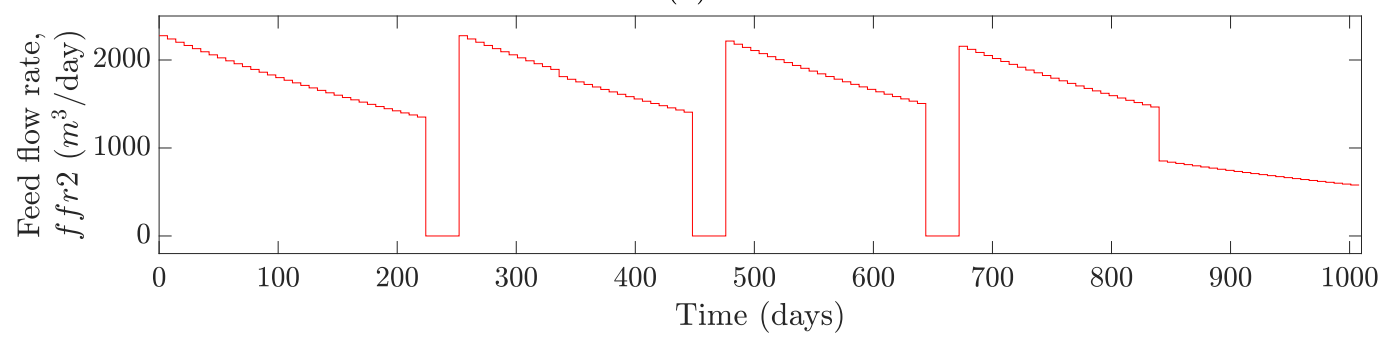

(c) Reactor 3

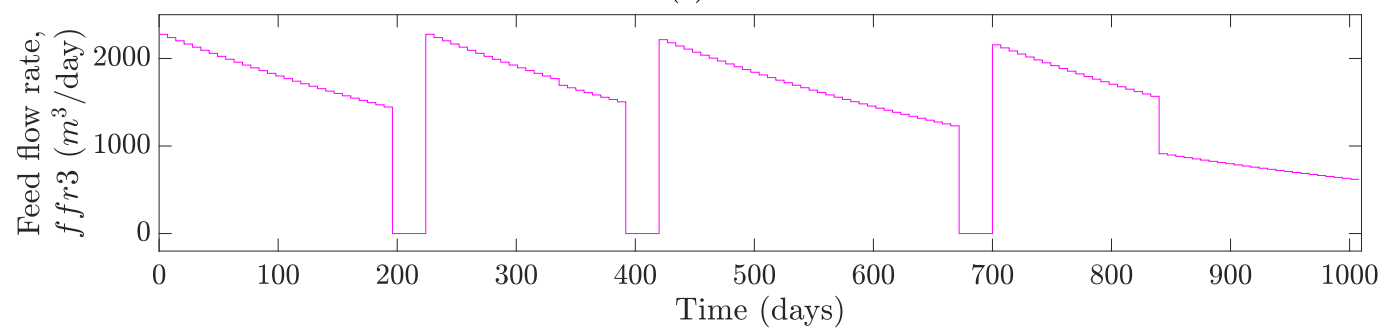

(d) Reactor 4

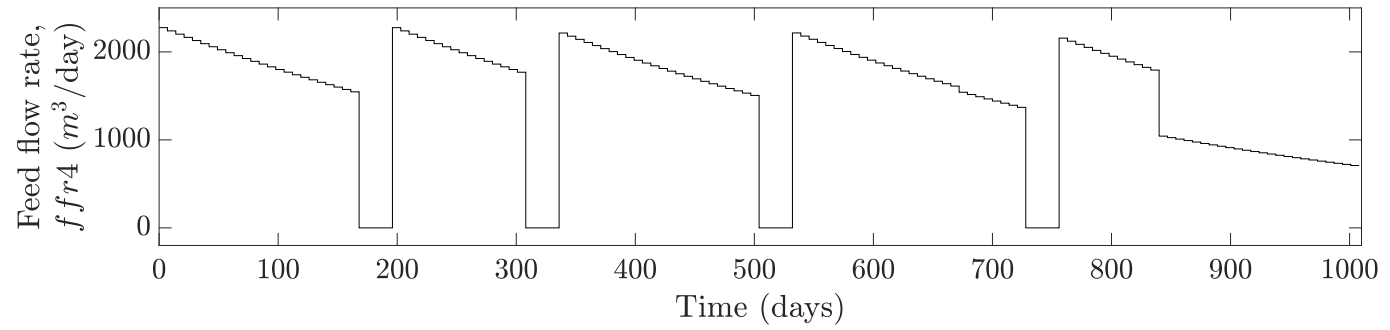

Figure 5: The variation of the feed flow rate over the time horizon in (a) Reactor 1 (b) Reactor 2 (c) Reactor 3 (d) Reactor 4. 


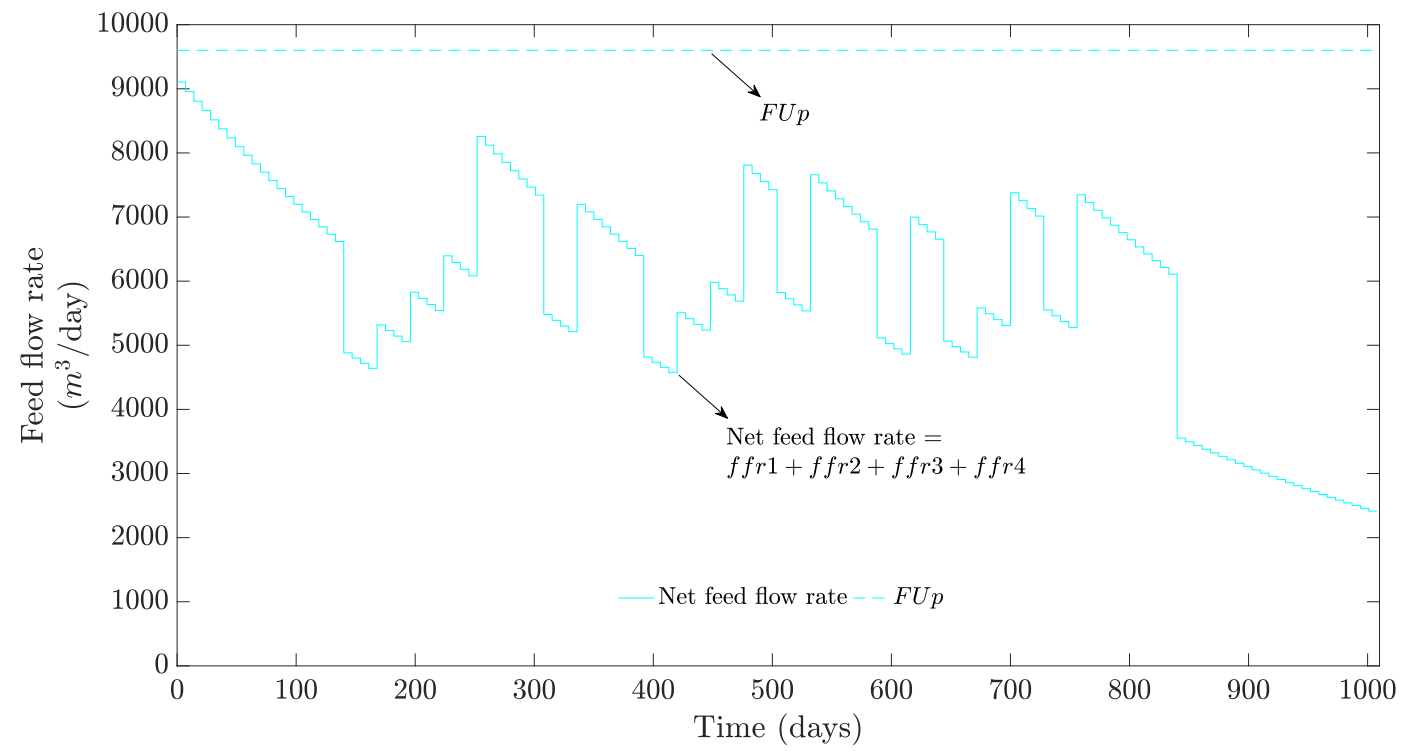

Figure 6: The variation of the net feed flow rate to the process over the time horizon.

(a)

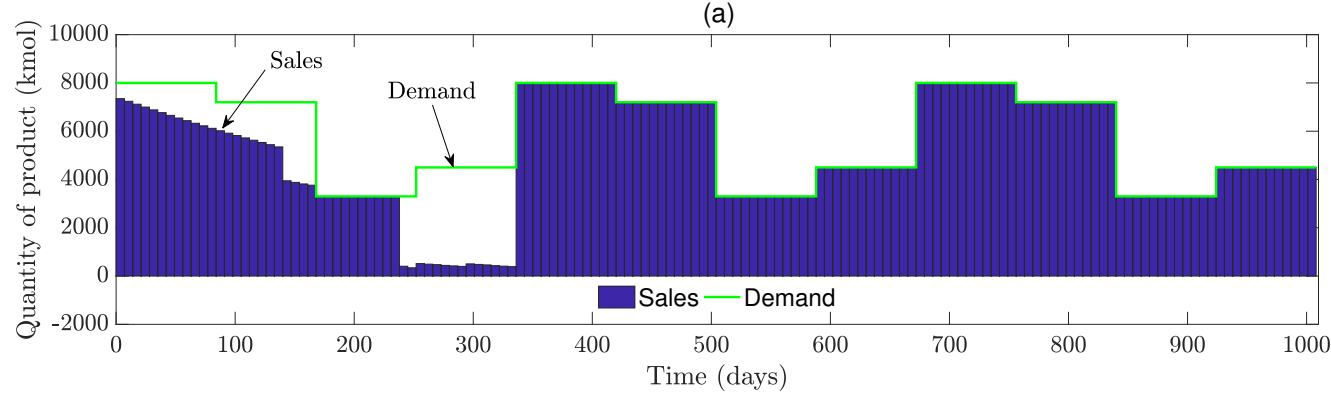

(b)

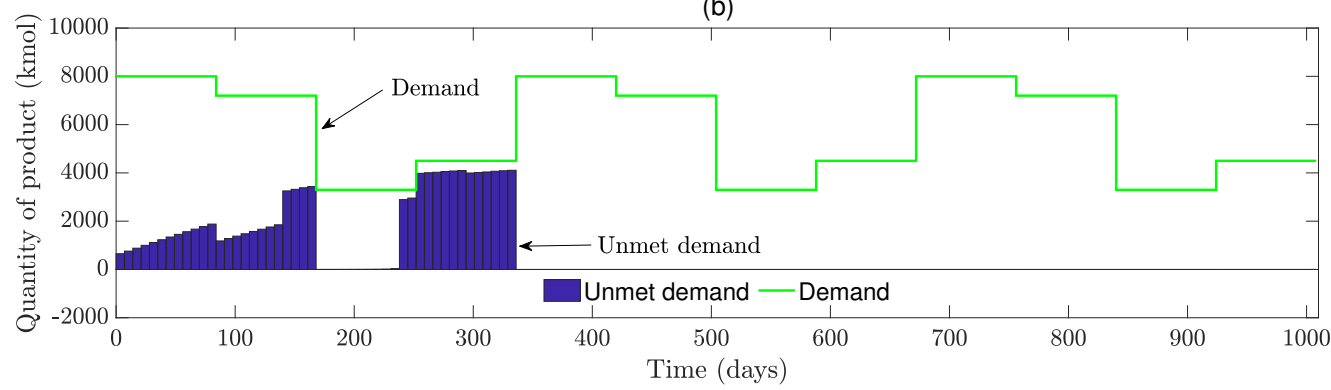

Figure 7: The variation of (a) sales and (b) unmet demand, in comparison to the demand over the whole time horizon. 
operation and a constant value at the starting catalyst activity during catalyst replacement follow directly from differential equations (11) and junction conditions (23).

The variation of the inventory level and cumulative inventory costs over the time horizon are shown in Figure 12. The oscillating behaviour of the inventory level follows from the interplay between the increase in inventory due to production from all reactors (differential equation (14)) and the decrease in inventory due to the sales (junction condition (25)). It is highlighted that towards the end of the first year, the inventory level shows a significant increase, despite there being a considerable amount of unmet demand at that time. This happens in order to enable greater amount of sales during later times when the product sales price has increased due to inflation, thereby enlarging the profit obtained. The trend for the cumulative inventory costs follows directly from differential equation (15) and junction condition (26).

The magnitudes of the various economic aspects that form the elements of the objective function are given in Table 6 . The table indicates that the cost of flow and raw material and the net penalty for unmet demand form the biggest proportions of the costs. The cost of catalyst changeovers contributes relatively less while the inventory costs form a very low percentage of the total expenditure. It is also seen that the costs of operation take away about $43.77 \%$ of the revenue generated by the product sales. 
(a) Reactor 1

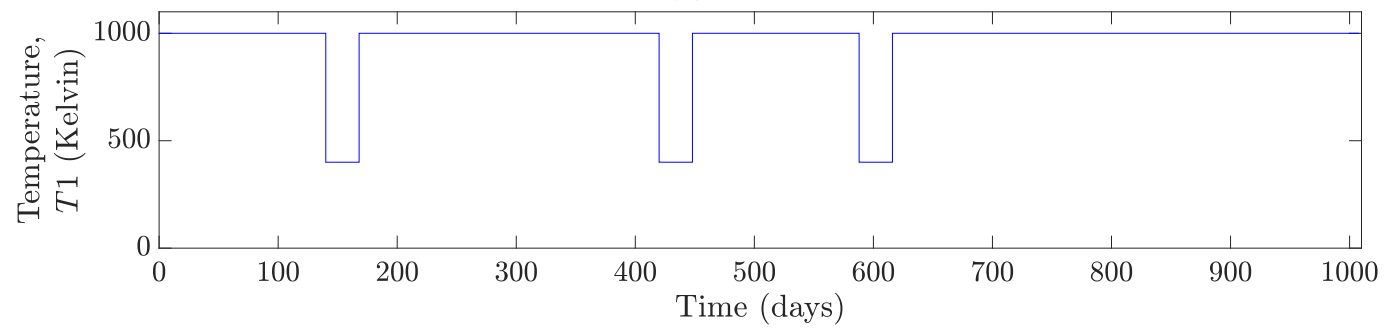

(b) Reactor 2

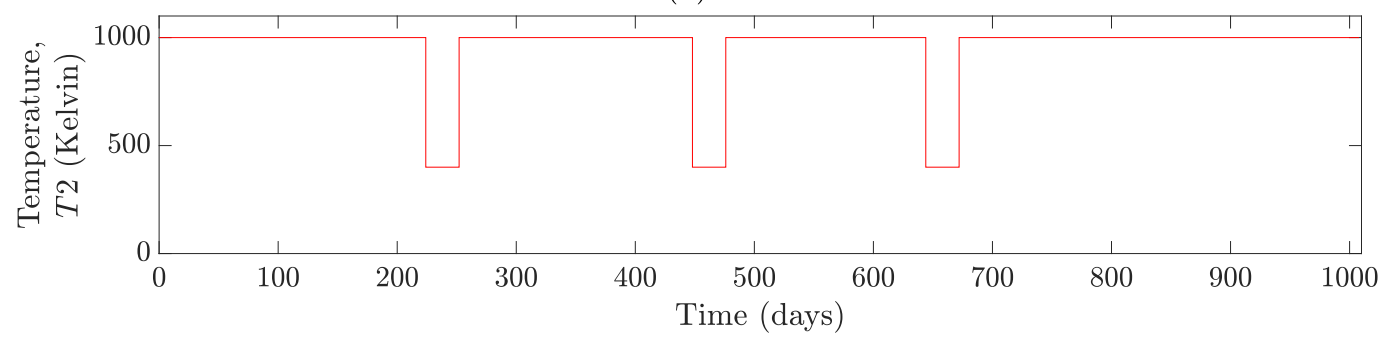

(c) Reactor 3

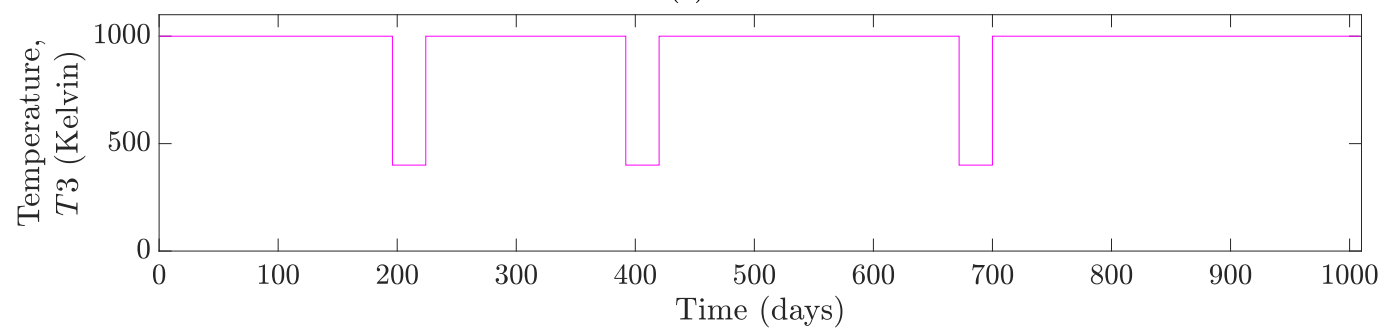

(d) Reactor 4

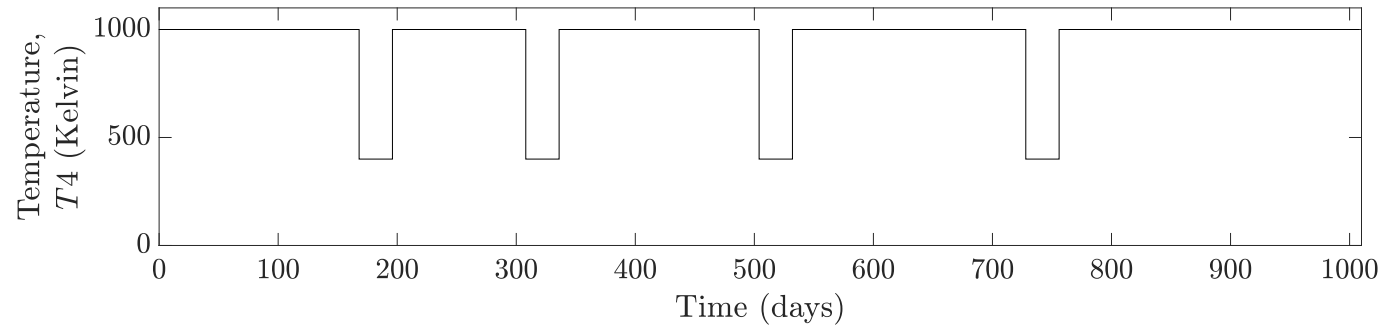

Figure 8: The variation of the temperature of operation over the time horizon in (a) Reactor 1 (b) Reactor 2 (c) Reactor 3 (d) Reactor 4. 
(a) Reactor 1

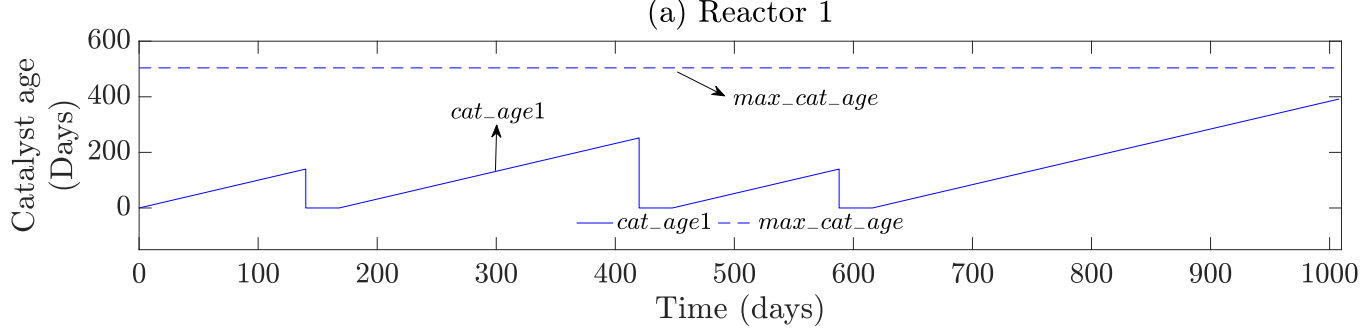

(b) Reactor 2

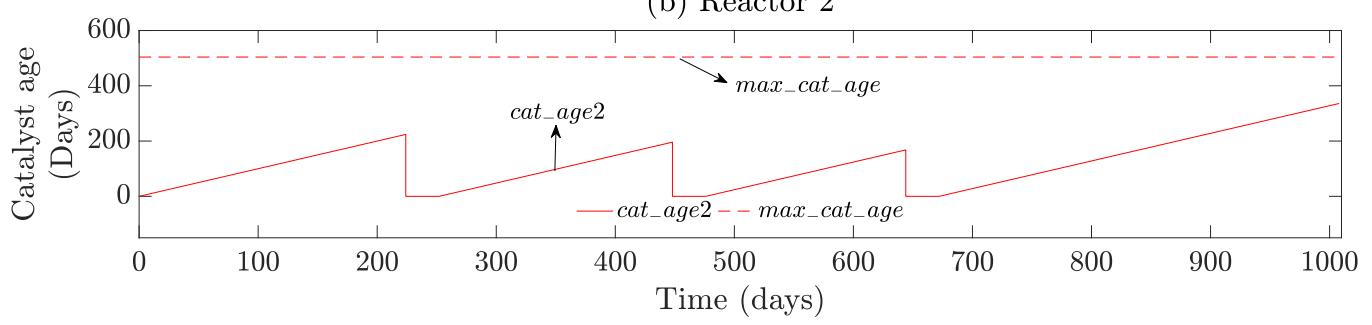

(c) Reactor 3

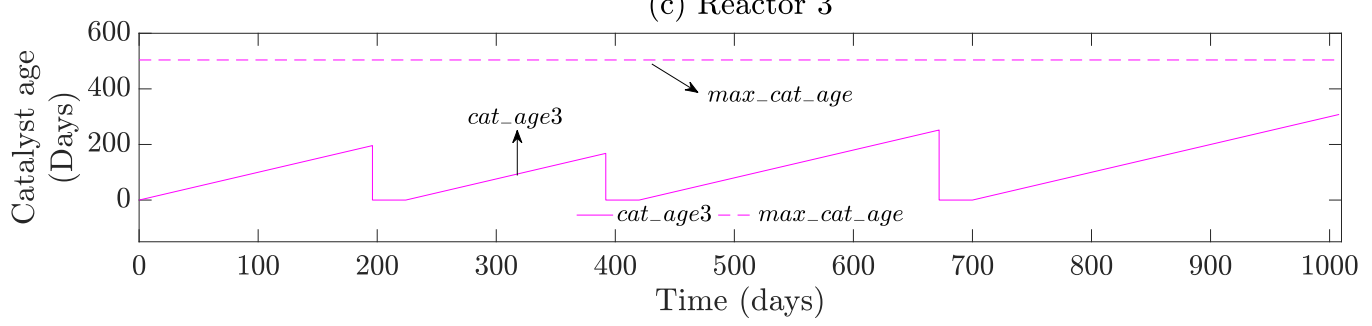

(d) Reactor 4

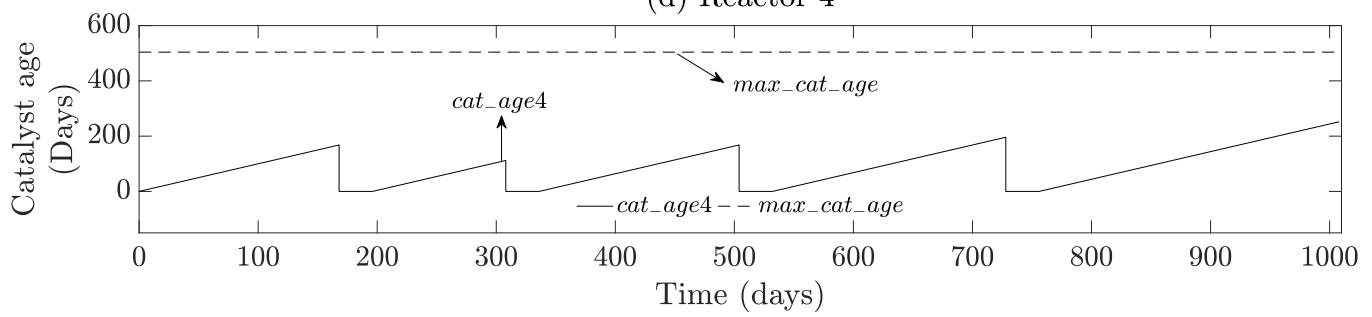

Figure 9: The variation of the catalyst age over the time horizon in (a) Reactor 1 (b) Reactor 2 (c) Reactor 3 (d) Reactor 4. 
(a) Reactor 1

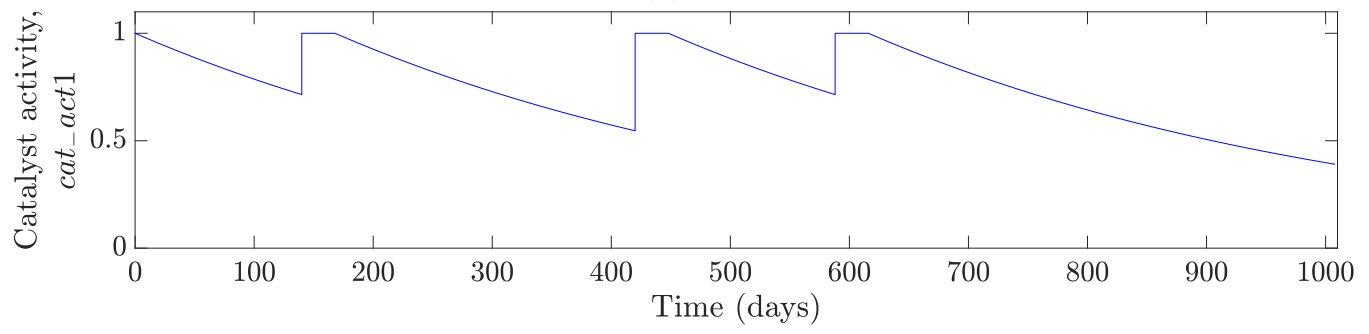

(b) Reactor 2

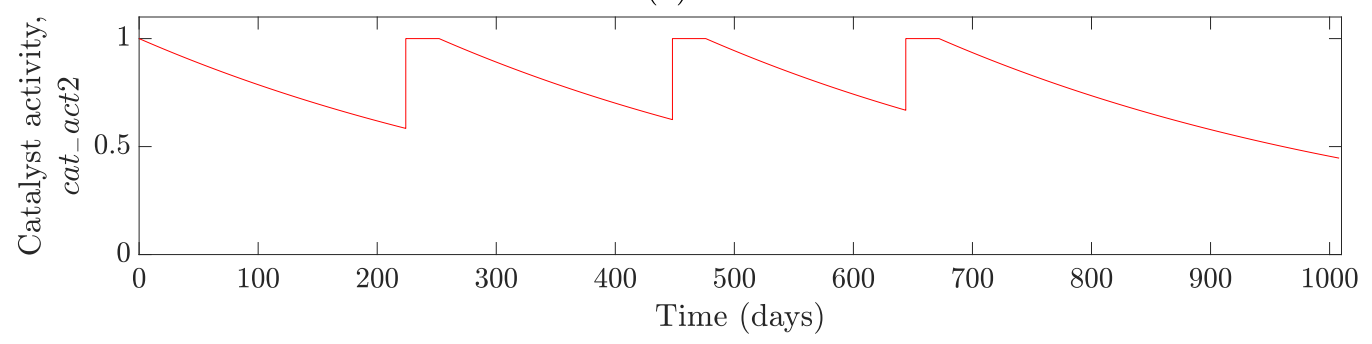

(c) Reactor 3

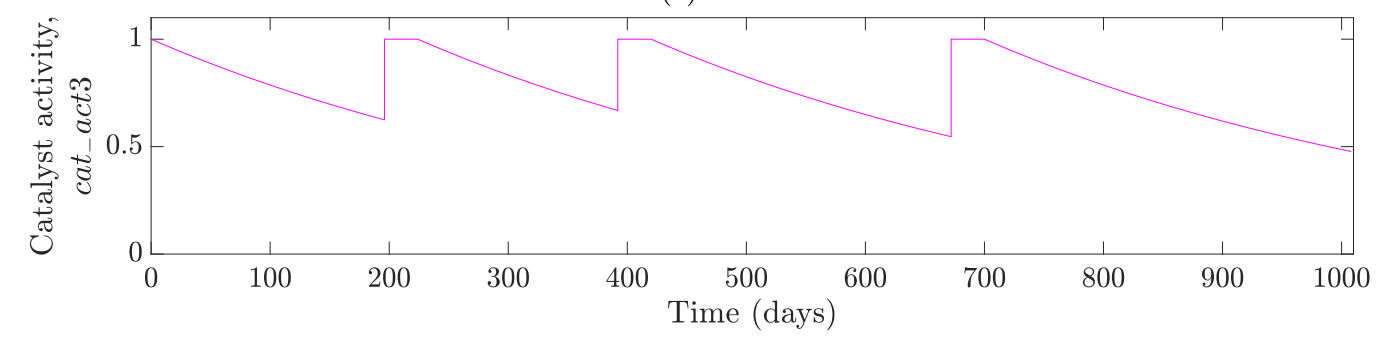

(d) Reactor 4

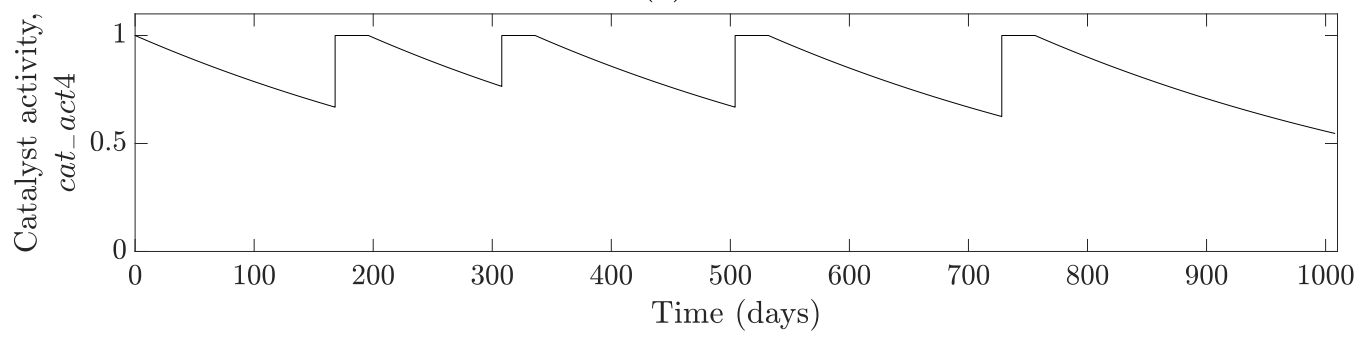

Figure 10: The variation of the catalyst activity over the time horizon in (a) Reactor 1 (b) Reactor 2 (c) Reactor 3 (d) Reactor 4. 
(a) Reactor 1

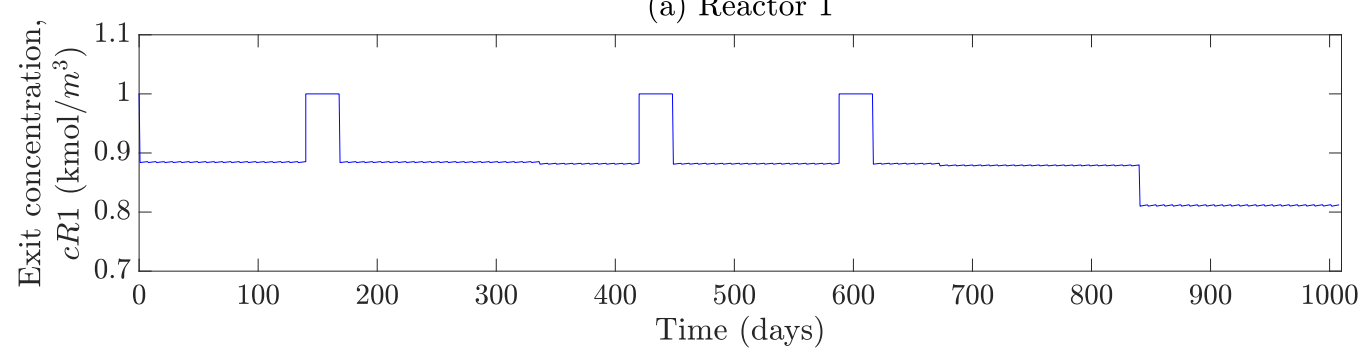

(b) Reactor 2

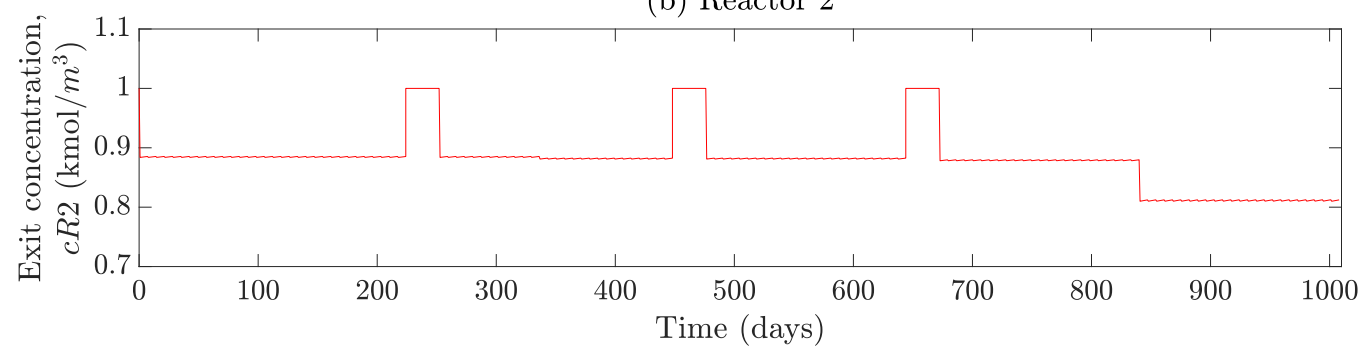

(c) Reactor 3

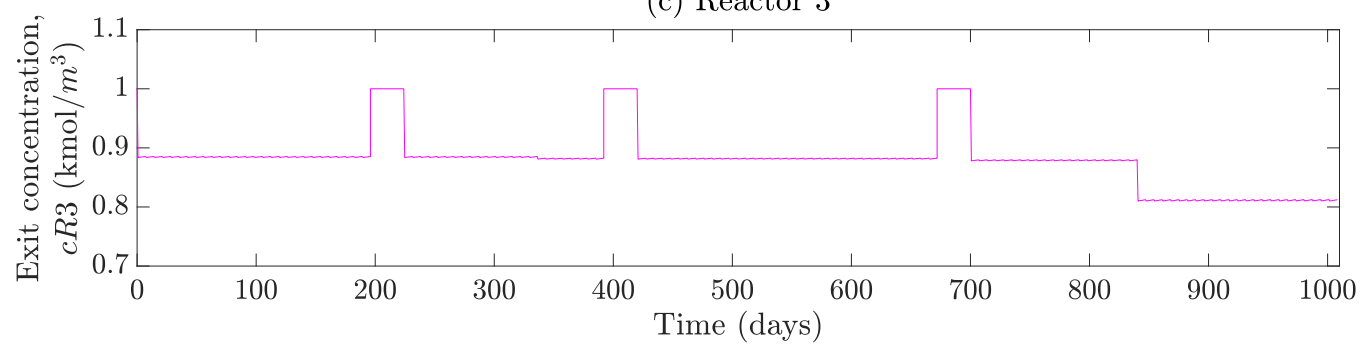

(d) Reactor 4

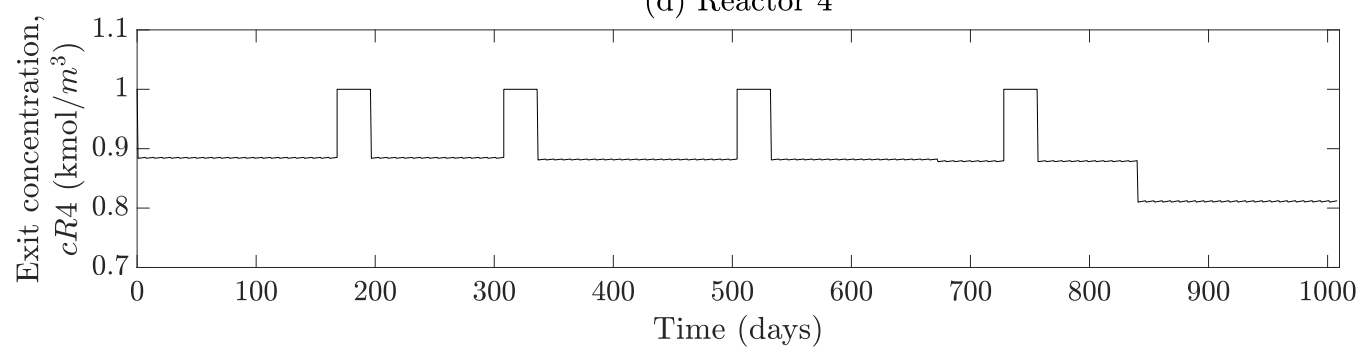

Figure 11: The variation of the reactant exit concentration over the time horizon in (a) Reactor 1 (b) Reactor 2 (c) Reactor 3 (d) Reactor 4. 


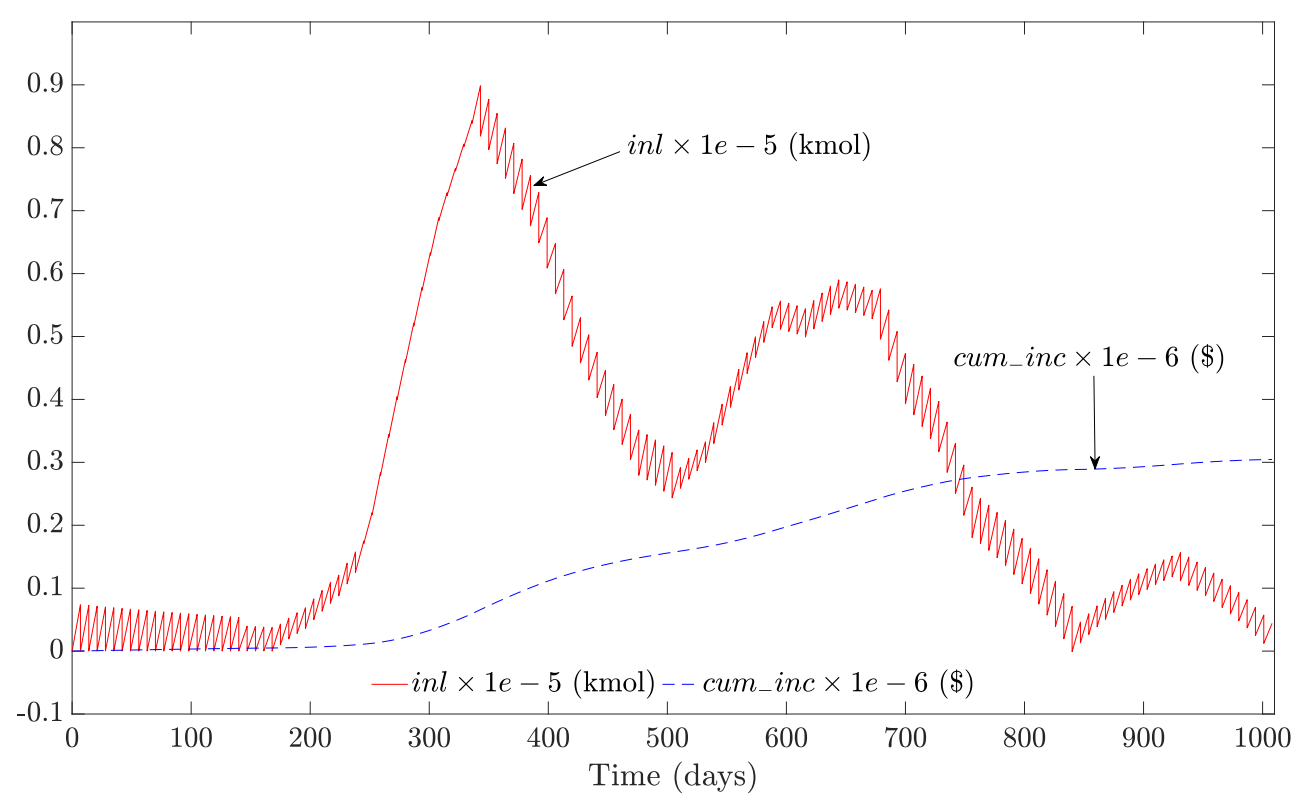

Figure 12: The variation of the product inventory level and cumulative inventory costs over the time horizon.

Table 6: Economic aspects of the best solution

\begin{tabular}{|c|c|c|c|}
\hline \multicolumn{2}{|c|}{ Economic aspect } & Symbol & Value (\$ Millions) \\
\hline \hline \multirow{4}{*}{ Costs } & Gross Revenue from Sales & $G R S$ & 774.672 \\
\cline { 2 - 4 } & Total Inventory Costs & $T I C$ & 0.305 \\
\cline { 2 - 4 } & Total Costs of Catalyst Changeovers & $T C C C$ & 33.762 \\
\cline { 2 - 4 } & Net Penalty for Unmet Demand & NPUD & 119.273 \\
\cline { 2 - 4 } & Total Flow Costs & $T F C$ & 185.737 \\
\hline \hline \multicolumn{2}{|c|}{ Profit } & $-N C$ & 435.595 \\
\hline
\end{tabular}




\section{Conclusions and further discussions}

In this paper, an optimal control approach for scheduling maintenance and production for processes using decaying catalysts, originally developed in a previous work by Adloor et al. (2020) for a single reactor, has been extended to parallel lines of reactors. This problem has been formulated as a multistage mixed-integer optimal control problem and the formulation has been applied to a case study of an industrial process that operates a single feed over a set of 4 parallel reactors to produce a single product.

The solution methodology used involves relaxing the binary restrictions on the catalyst changeover controls and solving a series of standard multistage optimal control problems using a feasible path approach, with a penalty term homotopy technique to enforce binary values for those controls. The highlights of this methodology are that the penalty term homotopy technique obviates the need for combinatorial optimisation methods to schedule catalyst changeovers and the feasible path approach guarantees accuracy, in addition to a smaller problem size that enables easier convergence to solutions.

Due to the non-convex nature of the problem, the optimisation was performed using 50 different initial guesses and each of these runs successfully converged to a local optimum without any difficulties. For the best solution among these runs, the profiles of the decision and state variables over the time horizon and the economics of the industrial process were presented. A notable result was that the operating policies for reactant exit concentration and temperature of each reactor correlated well with that of published literature (Szépe and Levenspiel, 1968) on the lab scale.

Ideally, a comparison of the solutions obtained with similar studies using

mixed-integer formulations should have been drawn. However, no previous 
publication exists that has explicitly considered the problem of optimising maintenance scheduling and production in parallel lines of reactors using decaying catalysts and so can be used for comparison. Even, a work by Schulz et al. (2006a), that formulated an MINLP model for an ethylene plant, while having similarities to the case study in this article, does not reveal the underlying equations and so the construction of a comparative study was not possible. It was because of these reasons that the case study considered here was created as a modification of a case study in Adloor et al. (2020) that considered a single reactor. It is important to stress the following points of comparison with mixed-integer techniques:

1. If used, the combinatorial nature of the mixed-integer methods can cause the computational effort involved in scheduling catalyst changeovers and ensuring conditions such as non-simultaneous catalyst replacement to become enormous, especially if the number of catalyst loads or the number of reactors increase. The problem size becomes so big that even obtaining a solution can become difficult. However, in the proposed methodology, catalyst replacement scheduling occurs without mixed-integer methods and conditions such as non-simultaneous replacement can be enforced using simple constraints. The nature of the methodology is such that an explosion in problem size is prevented and solutions can be obtained even if each reactor had an infinite number of catalyst loads available.

2. The use of state-of-the-art integrators in the feasible path approach enables solving even highly nonlinear differential equations to a high accuracy. But the mixed-integer methods can only handle such differential equations by discretising them under a steady state assumption. Thus, they cannot obtain an accurate description of the dynamics of the process.

3. The use of the feasible path approach implies the differential equations 
are solved without being considered as constraints in the optimisation phase. This enables a smaller problem size which facilitates convergence to optimal solutions. However, in mixed-integer methods, these differential equations would be discretised under a steady state assumption and imposed as equality constraints. The number of constraints in the problem thus become very large and this can cause difficulties in converging to optimal solutions.

4. The solution time using this methodology is quite large, in the order of days and is considerably higher than if mixed-integer techniques were to be used. This is because of the large computational effort spent in solving the differential equations to a high accuracy in each iteration of the optimisation. However, this is outweighed by the high quality of solutions obtained. Though not done here, solution times for this case and even larger problems can be greatly reduced, by exploiting the feature of scalability and making use of parallel computing and high performance computing facilities.

To conclude, the contributions of this paper are highlighted by the following advantages the optimal control approach has the potential to offer over mixed-integer methodologies for this problem:

1. It is robust because solutions can be obtained from any random initial guess, aided by the smaller number of constraints present.

2. It is reliable because solutions can be obtained to a high degree of accuracy using state-of-the-art integrators.

3. It is efficient because the catalyst replacements are scheduled inherently during the optimisation without using combinatorial optimisation methods.

The final points are with regard to the further applications of this methodology. The work here has considered a process with only a single feed pro- 
ducing a single product. However, in many real world applications, such as ethylene plants, parallel production lines process multiple feeds to produce multiple products and many publications have attempted to optimise maintenance scheduling and production in such set ups using mixed-integer programming methods. It would be important to apply the optimal control methodology to such problems, in order to overcome the drawbacks of the mixed-integer methods.

It is also sought to apply this methodology in plant wide optimisation involving upstream and downstream operations, interconnected by a complex network of reactors. In addition, robust decision making in industry requires considering the effect of parametric uncertainties in this problem and so, this is another area to be investigated in forthcoming work.

\section{Acknowledgements}

Author S.D. Adloor would like to acknowledge the Cambridge India Ramanujan Scholarship awarded by the Cambridge Trust and the Science and Engineering Research Board of India for the financial support of this research.

\section{Appendix A. Tables}


Table A.7: A table of the values of the catalyst changeover controls for all reactors, across all major iterations, for the best solution among the set of 50 runs. In this table, 'MI' is an abbreviation for Major Iteration.

\begin{tabular}{|c||c|c||c|c||c|c||c|c|}
\hline \multirow{2}{*}{ Month } & \multicolumn{3}{|c||}{$y 1$} & \multicolumn{2}{c||}{$y 2$} & \multicolumn{2}{c||}{$y 3$} & \multicolumn{2}{c|}{$y 4$} \\
\cline { 2 - 9 } & MI 1 & MI 2 & MI 1 & MI 2 & MI 1 & MI 2 & MI 1 & MI 2 \\
\hline \hline 1 & 1 & 1 & 1 & 1 & 1 & 1 & 1 & 1 \\
\hline 2 & 1 & 1 & 1 & 1 & 1 & 1 & 1 & 1 \\
\hline 3 & 1 & 1 & 1 & 1 & 1 & 1 & 1 & 1 \\
\hline 4 & 1 & 1 & 1 & 1 & 0.79 & 1 & 1 & 1 \\
\hline 5 & 1 & 1 & 0.76 & 1 & 1 & 1 & 1 & 1 \\
\hline 6 & 0.52 & 0 & 1 & 1 & 1 & 1 & 1 & 1 \\
\hline 7 & 1 & 1 & 1 & 1 & 1 & 1 & 0.48 & 0 \\
\hline 8 & 1 & 1 & 1 & 1 & 1 & 0 & 1 & 1 \\
\hline 9 & 1 & 1 & 0.53 & 0 & 0.47 & 1 & 1 & 1 \\
\hline 10 & 1 & 1 & 1 & 1 & 1 & 1 & 1 & 1 \\
\hline 11 & 0.59 & 1 & 1 & 1 & 1 & 1 & 1 & 1 \\
\hline 12 & 1 & 1 & 1 & 1 & 1 & 1 & 0.46 & 0 \\
\hline 13 & 1 & 1 & 0.88 & 1 & 1 & 1 & 1 & 1 \\
\hline 14 & 1 & 1 & 1 & 1 & 1 & 1 & 1 & 1 \\
\hline 15 & 1 & 1 & 1 & 1 & 0.50 & 0 & 1 & 1 \\
\hline 16 & 0.50 & 0 & 1 & 1 & 1 & 1 & 1 & 1 \\
\hline 17 & 1 & 1 & 0.42 & 0 & 1 & 1 & 1 & 1 \\
\hline 18 & 1 & 1 & 1 & 1 & 1 & 1 & 1 & 1 \\
\hline 19 & 1 & 1 & 1 & 1 & 1 & 1 & 0.43 & 0 \\
\hline 20 & 1 & 1 & 1 & 1 & 0.59 & 1 & 1 & 1 \\
\hline 21 & 1 & 1 & 0.90 & 1 & 1 & 1 & 1 & 1 \\
\hline 22 & 0.40 & 0 & 1 & 1 & 1 & 1 & 1 & 1 \\
\hline 23 & 1 & 1 & 1 & 1 & 1 & 1 & 0.82 & 1 \\
\hline 24 & 1 & 1 & 1 & 0 & 1 & 1 & 1 & 1 \\
\hline 25 & 1 & 1 & 0.47 & 1 & 0.53 & 0 & 1 & 1 \\
\hline 26 & 1 & 1 & 1 & 1 & 1 & 1 & 1 & 1 \\
\hline 27 & 1 & 1 & 1 & 1 & 1 & 1 & 0.67 & 0 \\
\hline 28 & 0.81 & 1 & 1 & 1 & 1 & 1 & 1 & 1 \\
\hline 29 & 1 & 1 & 1 & 1 & 1 & 1 & 1 & 1 \\
\hline 30 & 1 & 1 & 1 & 1 & 1 & 1 & 1 & 1 \\
\hline 31 & 1 & 1 & 0.82 & 1 & 0.81 & 1 & 1 & 1 \\
\hline 32 & 0.87 & 1 & 1 & 1 & 1 & 1 & 0.90 & 1 \\
\hline 33 & 1 & 1 & 1 & 1 & 1 & 1 & 1 & 1 \\
\hline 34 & 1 & 1 & 1 & 1 & 1 & 1 & 1 & 1 \\
\hline 35 & 1 & 1 & 1 & 1 & 1 & 1 & 1 & 1 \\
\hline 36 & 1 & 1 & 1 & 1 & 1 & 1 & 1 & 1 \\
\hline & & & & & & & & \\
\hline
\end{tabular}




\section{References}

Adloor, S., Pons, T., Vassiliadis, V., 2020. An optimal control approach to scheduling and production in a process using decaying catalysts. Computers \& Chemical Engineering 135, 106743.

Alanqar, A., Durand, H., Albalawi, F., Christofides, P.D., 2016. Integrating production scheduling and process operation via economic model predictive control, in: 2016 IEEE 55th Conference on Decision and Control (CDC), IEEE. pp. 3190-3195.

Alanqar, A., Durand, H., Albalawi, F., Christofides, P.D., 2017. An economic model predictive control approach to integrated production management and process operation. AIChE Journal 63, 1892-1906.

Andersson, J., 2013. A General-Purpose Software Framework for Dynamic Optimisation. PhD thesis. Arenberg Doctoral School, KU Leuven. Department of Electrical Engineering (ESAT/SCD) and Optimisation in Engineering Center, Kasteelpark Arenberg 10, 3001-Heverlee, Belgium.

Castro, P.M., Grossmann, I.E., Veldhuizen, P., Esplin, D., 2014. Optimal maintenance scheduling of a gas engine power plant using generalised disjunctive programming. AIChE journal 60, 2083-2097.

Ellis, M., Liu, J., Christofides, P.D., 2017. Economic model predictive control. volume 2. Springer.

Heluane, H., Colombo, M., Hernández, M., Graells, M., Puigjaner, L., 2007. Enhancing sugar cane process performance through optimal production scheduling. Chemical Engineering and Processing: Process Intensification 46, 198-209.

Heluane, H., Colombo, M.A., Hernandez, M.R., Sequei ra, S.E., Graells, M., Puigjaner, L., 2004. Scheduling of continuous parallel lines in the evapora- 
tion section of sugar plants. Chemical Engineering Communications 191, $1121-1146$.

Hindmarsh, A.C., Brown, P.N., Grant, K.E., Lee, S.L., Serban, R., Shumaker, D.E., Woodward, C.S., 2005. SUNDIALS: Suite of nonlinear and differential/algebraic equation solvers. ACM Transactions on Mathematical Software (TOMS) 31, 363-396.

Jain, V., Grossmann, I.E., 1998. Cyclic scheduling of continuous parallelprocess units with decaying performance. AIChE Journal 44, 1623-1636.

Jin, Y., Li, J., Du, W., Qian, F., 2015. Integrated operation and cyclic scheduling optimisation for an ethylene cracking furnaces system. Industrial \& Engineering Chemistry Research 54, 3844-3854.

Kopanos, G.M., Xenos, D.P., Cicciotti, M., Pistikopoulos, E.N., Thornhill, N.F., 2015. Optimisation of a network of compressors in parallel: Operational and maintenance planning-the air separation plant case. Applied Energy 146, 453-470.

Lim, H., Choi, J., Realff, M., Lee, J.H., Park, S., 2006. Development of optimal decoking scheduling strategies for an industrial naphtha cracking furnace system. Industrial \& Engineering Chemistry Research 45, 57385747.

Lin, Y., Du, W., 2018. A two-level optimisation framework for cyclic scheduling of ethylene cracking furnace system, in: 2018 IEEE Congress on Evolutionary Computation (CEC), IEEE. pp. 1-8.

Liu, C., Zhang, J., Xu, Q., Li, K., 2010. Cyclic scheduling for best profitability of industrial cracking furnace system. Computers \& Chemical Engineering 34, 544-554. 
Liu, S., Yahia, A., Papageorgiou, L.G., 2014. Optimal production and maintenance planning of biopharmaceutical manufacturing under performance decay. Industrial \& Engineering Chemistry Research 53, 17075-17091.

McKay, M.D., Beckman, R.J., Conover, W.J., 1979. Comparison of three methods for selecting values of input variables in the analysis of output from a computer code. Technometrics 21, 239-245.

Sager, S., 2005. Numerical methods for mixed-integer optimal control problems. Der Andere Verlag Tönning.

Sager, S., 2009. Reformulations and algorithms for the optimisation of switching decisions in nonlinear optimal control. Journal of Process Control 19, $1238-1247$.

Schulz, E.P., Bandoni, J.A., Diaz, M.S., 2006a. Optimal shutdown policy for maintenance of cracking furnaces in ethylene plants. Industrial \& Engineering Chemistry Research 45, 2748-2757.

Schulz, E.P., Bandoni, J.A., Diaz, M.S., 2006b. Process optimisation and scheduling of parallel furnaces shutdown in large-scale plants, in: Computer Aided Chemical Engineering. Elsevier. volume 21, pp. 1833-1838.

Su, L., Tang, L., Grossmann, I.E., 2015. Computational strategies for improved MINLP algorithms. Computers \& Chemical Engineering 75, 40-48.

Su, L., Tang, L., Grossmann, I.E., 2016. Scheduling of cracking production process with feedstocks and energy constraints. Computers \& Chemical Engineering 94, 92-103.

Szépe, S., Levenspiel, O., 1968. Optimal temperature policies for reactors subject to catalyst deactivation - Batch reactor. Chemical Engineering Science 23, 881-894. 
Vassiliadis, V.S., 1993. Computational solution of dynamic optimisation problems with general differential-algebraic constraints. Ph.D. thesis. University of London, London, England.

Vassiliadis, V.S., Sargent, R., Pantelides, C.C., 1994a. Solution of a class of multistage dynamic optimisation problems. 1. Problems without path constraints. Industrial \& Engineering Chemistry Research 33, 2111-2122.

Vassiliadis, V.S., Sargent, R.W., Pantelides, C.C., 1994b. Solution of a class of multistage dynamic optimisation problems. 2. Problems with path constraints. Industrial \& Engineering Chemistry Research 33, 2123-2133.

Wächter, A., Biegler, L.T., 2006. On the implementation of an interiorpoint filter line-search algorithm for large-scale nonlinear programming. Mathematical Programming 106, 25-57.

Wang, Z., Li, Z., Feng, Y., Rong, G., 2016. Integrated short-term scheduling and production planning in an ethylene plant based on Lagrangian decomposition. The Canadian Journal of Chemical Engineering 94, 1723-1739.

Xenos, D.P., Kopanos, G.M., Cicciotti, M., Thornhill, N.F., 2016. Operational optimisation of networks of compressors considering condition-based maintenance. Computers \& Chemical Engineering 84, 117-131.

Yu, K., While, L., Reynolds, M., Wang, X., Wang, Z., 2017. Cyclic scheduling for an ethylene cracking furnace system using diversity learning teachinglearning-based optimisation. Computers \& Chemical Engineering 99, 314324 .

Zhao, C., Liu, C., Xu, Q., 2010. Cyclic scheduling for ethylene cracking furnace system with consideration of secondary ethane cracking. Industrial \& Engineering Chemistry Research 49, 5765-5774. 